Kilian Spandler

\title{
Regionale Integration durch normative Diskurse
}

\author{
Die Legitimierung von Verrechtlichungsprozessen in der ASEAN und der
} EU

Der Beitrag argumentiert, dass die variierende Erklärungskraft klassischer Theorien regionaler Integration auf normativ eingebettete Diskursdynamiken zurückzuführen ist. Grundlegend für diese These ist ein Modell regionaler Integration, das Anleihen bei Theorien kommunikativen und strategischen Handelns einerseits und der English School andererseits nimmt. Im Gegensatz zu den rationalistischen Frameworks von Intergouvernementalismus und Neofunktionalismus stellt es die diskursive Verfasstheit von Integration in den Vordergrund. Dementsprechend begreife ich konkurrierende Integrationslogiken nicht als objektive Kausalmechanismen, sondern als Diskursmotive. Deren Resonanz in konkreten Aushandlungsprozessen von institutionellem Wandel wird von einem normativen Kontext bedingt, der sich aus regionalen Primärinstitutionen konstituiert. Durch eine vergleichende Studie von Verrechtlichungsprozessen in der Europäischen Union (EU) und der Association of Southeast Asian Nations (ASEAN) zeigt der Beitrag die variable Bedeutung funktionalistischer frames für institutionellen Wandel in Regionalorganisationen und erweitert so unser Verständnis der 'scope conditions' klassischer Theorien. Damit schlägt er eine Brücke zwischen der Europäischen Integrations- und der Vergleichenden Regionalismusforschung.

\section{Einleitung*}

Die klassischen Ansätze des Intergouvernementalismus und Neofunktionalismus spielen trotz des wachsenden Theoriepluralismus in der Literatur zur Erklärung regionaler Integration nach wie vor eine zentrale Rolle. Seit den Anfängen der Integrationsforschung in den 1950er-Jahren haben sie sich in produktiver Auseinandersetzung mit konkurrierenden Ideen weiterentwickelt und dadurch zumindest im Kontext der Europäischen Union (EU) ihre Relevanz bewahrt (Hooghe/Marks 2019: 1113-1114). Beiden Ansätzen liegt eine funktional-institutionalistische Erklärungslogik zugrunde, der zufolge strukturelle Nachfragefaktoren, vermittelt durch bestimmte politische Mechanismen, die institutionelle Entwicklung von Regionalorganisationen vorantreiben. Der Hauptunterschied liegt in den jeweils als zentral identifizierten Akteuren und Feedbackprozessen. Während der Intergouvernementalismus zwischenstaatliche Verhandlungen als zentralen Transmissionsriemen

* Ich danke Maria Debre, Marisa Irawan, Vera van Hüllen, den TeilnehmerInnen der Ersten Jahrestagung des DVPW-Arbeitskreises Europa- und Regionalismusforschung (Magdeburg, 2.-3. Mai 2019) und den anonymen GutachterInnen für ihre hilfreichen Kommentare. 
sieht, rückt der Neofunktionalismus spillover-Prozesse und Pfadabhängigkeiten ins Zentrum und betont den zunehmenden Kontrollverlust staatlicher Akteure über den Integrationsprozess (Schimmelfennig 2018).

Die Vergleichende Regionalismusforschung, welche seit etwa einem Jahrzehnt als eigenständiges Feld der Internationalen Beziehungen (IB) auftritt, hat diese klassischen Erklärungsmuster als EU-zentristisch zurückgewiesen (Acharya 2012a: 6-8; Murray 2010: 317-320; Sbragia 2008: 30-31). Sie verweist dazu unter anderem auf empirische Evidenz aus anderen Weltregionen, die dem postulierten kausalen Zusammenhang zwischen funktionaler Integrationsnachfrage nichtstaatlicher und staatlicher Akteure einerseits und institutionellem Wandel andererseits infrage stellen (Börzel 2016: 50-51; Lenz/Marks 2016: 519). VordenkerInnen ${ }^{1}$ der klassischen Theorien haben dies freilich früh erkannt und Kontextbedingungen spezifiziert, die ihren Hypothesen im europäischen Fall besondere Durchschlagskraft verleihen (Haas 1961: 377-378). Die Vergleichende Regionalismusforschung hat sich unterdessen von den klassischen Integrationstheorien emanzipiert und Ansätze und Konzepte in den Mittelpunkt ihrer Analysen gestellt, die den spezifischen Motiven und Kontextbedingungen von Regionalismus außerhalb Europas besser gerecht werden sollen. ${ }^{2}$ Dies ist keine rein akademische Abgrenzung, ist sie doch oft auch von der Suche nach Alternativen zu den hegemonialen Integrationsmodellen motiviert, die die EU in ihren Außenbeziehungen auf andere Regionen projiziert (Acharya 2016: 109-110).

In diesem Beitrag argumentiere ich, dass die zentralen Ideen der klassischen Integrationstheorien durchaus für die Vergleichende Regionalismusforschung fruchtbar gemacht werden können. Die Voraussetzung dafür ist eine fundamentale Umdeutung, die klassische Integrationslogiken wie z.B. den Funktionalismus nicht als objektive Kausalmechanismen begreift, sondern als diskursive frames endogenisiert und sie dadurch aus ihrem rationalistischen Theoriekontext herauslöst. Grundlegend für diese Interpretation ist ein Modell regionaler Integration, das Anleihen bei Theorien kommunikativen und strategischen Handelns einerseits (Schimmelfennig 2003) und der English School (Buzan 2004) andererseits nimmt. Zwei Kernargumente tragen diesen Ansatz: Erstens sind regionale Integrationsprozesse maßgeblich diskursiv verfasst, d.h. sie werden von regionalen Akteuren argumentativ ausgehandelt. Letztere können auf verschiedene legitimierende frames zurückgreifen, um konkrete institutionelle Formen zu rechtfertigen oder zu delegitimieren. Zweitens wird die Resonanz solcher frames von einem normativen Kontext bedingt, der sich - in der Terminologie der English School - aus regionalen Primärinstitutionen konstituiert (Buzan 2004: 161-204). Ob beispielsweise eine funktionalistische Integrationslogik von Bedeutung für institutionellen Wandel in spezifischen Regional-

1 Der Einfachheit halber verwende ich in diesem Text inklusive Genderformen für alle Gruppen, auch wenn sich deren Mitglieder in konkreten Fällen unter Umständen einer einheitlichen Geschlechtsidentität zuschreiben lassen.

2 Manche AutorInnen gehen dabei soweit, den Begriff der Integration selbst als EU-zentristisch zurückzuweisen und ihn mit einem grundsätzlich an staatlicher Autonomie orientierten Regionalismus im Globalen Süden zu kontrastieren (Acharya 2016: 116-117). 
organisationen ist, hängt damit nicht wie in den klassischen Theorien von objektiven strukturell-materiellen Kontextfaktoren ab, sondern von der Wahl entsprechender Argumente durch politische Eliten und ihrer erfolgreichen Rückbindung an den primärinstitutionellen Kontext.

Durch eine vergleichende Studie von Institutionalisierungsprozessen in den regionalen Rechtsordnungen der EU und der Association of Southeast Asian Nations (ASEAN) zeigt der Artikel, inwiefern dieser Ansatz die variable Bedeutung der funktionalistischen Integrationslogik erklären kann. Drei Aspekte machen diesen Vergleich aus theoretischer Sicht besonders interessant. Erstens ist die Forschung zur rechtlichen Integration der EU ganz besonders von der Auseinandersetzung zwischen intergouvernementalen und neofunktionalistischen Ansätzen geprägt (siehe Abschnitt 2). Aufgrund der Dominanz funktional-institutionalistischer Ansätze eignet sich das Feld besonders als hard case für eine Plausibilitätsprüfung des Arguments, dass normative Diskurse zentral für den spezifischen Verlauf regionaler Integration sind. Zweitens dominiert in politikwissenschaftlichen Studien der europäischen Rechtsordnung spätestens seit dem Vertrag von Maastricht das SuiGeneris-Paradigma, wonach die EU zwar kein Verfassungsstaat im traditionellen Sinne ist, sich aber eben auch essenziell von herkömmlichen Regionalorganisationen unterscheidet. Dementsprechend sind Vergleiche der EU mit anderen Regionen gerade in diesem Politikbereich quasi nichtexistent. Es drängt sich aber die Frage auf, ob sich die EU-Forschung durch diese idiographische Fokussierung nicht das theoriekritische und - bildende Potenzial komparativer Perspektiven entgehen lässt. Drittens ist die weitgehende Abwesenheit neofunktionalistischer Deutungen der rechtlichen Institutionalisierung in der ASEAN ein klares Indiz für die begrenzte Übertragbarkeit klassischer Erklärungsansätze auf nichteuropäische Kontexte. In der Tat blieb die Verrechtlichung der ASEAN trotz der Allgegenwärtigkeit funktionalistischer Argumente im Diskurs um die ASEAN-Charta stark begrenzt. Die von den klassischen Theorien postulierten Mechanismen greifen in diesem Fall offenbar nicht oder zumindest nicht mit der gleichen Effektivität wie in der EU.

Die Umdeutung der funktionalistischen Integrationslogik von einem kausalen Mechanismus zu einem diskursiven Motiv untermauert das Argument, dass die Vergleichende Regionalismusforschung Ansätze aus der Europäischen Integrationsforschung nutzbar machen kann, ohne den vermeintlichen Eurozentrismus ihrer epistemologischen und theoretischen Annahmen sowie deren Implikationen zu importieren. Gleichzeitig kann die Untersuchung normativer Resonanzbedingungen für verschiedene Argumente durch Berücksichtigung ihres primärinstitutionellen Kontexts die Erklärungskraft klassischer Ansätze erhöhen, indem sie zu einer genaueren Definition ihrer normativen scope conditions beiträgt. In diesem Sinne unterstützt der Artikel Forderungen nach einem kritischen, aber produktiven Dialog zwischen der Europäischen Integrations- und der Vergleichenden Regionalismusforschung (Sbragia/Söderbaum 2010: 579-580; Warleigh-Lack 2006: 751).

Der folgende Abschnitt skizziert in Kürze die Kritik der Vergleichenden Regionalismusforschung an den klassischen Integrationstheorien. In Abschnitt 3 führe ich das Konzept des normativen Diskurses ein und interpretiere unterschiedliche Inte- 
grationslogiken als frames zur Legitimierung von institutionellem Wandel in Regionalorganisationen. Unter Anwendung dieses analytischen Frameworks vergleicht Abschnitt 4 die rechtliche Integration der EU und der ASEAN jeweils mit Fokus auf eine Kernphase - die auf die Maastrichter Verträge hinführenden Debatten zur Unionsbürgerschaft einerseits und die in der ASEAN-Charta kulminierenden Diskurse zur Reform der Streitbeilegungsmechanismen andererseits.

\section{Klassische Integrationstheorien und Vergleichende Regionalismusforschung}

Die nach der Jahrtausendwende einsetzende schrittweise Entwicklung der Vergleichenden Regionalismusforschung als eigenständiges Feld der Internationalen Beziehungen (IB) erfolgte von Beginn an in einer kritischen Auseinandersetzung mit den klassischen Integrationstheorien, die aus der europäischen Erfahrung hervorgegangen waren (Söderbaum 2016: 29-33). ${ }^{3}$ Ein Hauptkritikpunkt war dabei die Dominanz utilitaristischer Erklärungsmuster. Die zentrale Annahme neofunktionalistischer Ansätze ist, dass initiale regionale Integration eine Nachfrage vor allem nichtstaatlicher Akteure nach weiteren supranationalen Politiklösungen generiert. Diese übersetzt sich durch verschiedene spillover-Effekte in progressive Institutionalisierung (Haas 1958: 291-299; Schmitter 1969: 162-164). Das Resultat ist eine Präferenzkonstellation politisch einflussreicher Akteure, die die ursprüngliche intergouvernementale Kontrolle des Vergemeinschaftungsprozesses unterwandert und eine inkrementelle Supranationalisierung befördert. Der Intergouvernementalismus führt institutionelle Entwicklungen ebenfalls auf Nachfragefaktoren wie Wirtschaftsoder Sicherheitsinterdependenzen zurück. Er behauptet lediglich im Gegensatz zum Neofunktionalismus die fortwährende Kontrolle staatlicher Akteure über die Intensität der Integration, die diese auf der Grundlage exogen stabiler Präferenzen in einer Abwägung von Souveränitätskosten und Kooperationsgewinnen bestimmen wobei sie in der liberalen Variante in erster Linie als Vertreter innerstaatlicher Interessengruppen agieren (Moravcsik 1998: 3).

Trotz unterschiedlicher Annahmen in Bezug auf dominante Akteure und deren Präferenzen sowie die zentralen Feedbackeffekte beruhen beide klassische Integrationstheorien letztendlich auf funktional-institutionalistischen Erklärungsmustern. Integration folgt demnach der funktionalen Nachfrage nach Politiklösungen jenseits des Nationalstaats, die sich mittels verschiedener Kausalmechanismen in institutionellen Wandel übersetzt (Schimmelfennig 2012: 397). Diese Gemeinsamkeiten zeigen sich auch in der Deutung rechtlicher Integration in der EU. Dem neofunktiona-

3 Ich verwende den Begriff klassisch hier nicht als Epochenbezeichnung, sondern schließe auch jüngere Ansätze ein, die in der Theorietradition der institutionalistischen Integrationsforschung der 1950er- und 1960er-Jahre stehen. Einen tiefergehenden Überblick über die Theorielandschaft bieten unter anderem Schimmelfennig (2018), Bieling und Lerch (2012) sowie Wiener et al. (2018). Auf den Postfunktionalismus als jüngeren Ansatz der EU-Integrationsforschung (Hooghe/Marks 2009) gehe ich nur am Rande ein, da er in der Vergleichenden Regionalismusforschung bisher nicht rezipiert wurde. 
listischen Narrativ zufolge ist die Emergenz einer Europäischen Rechtsordnung auf das strategische Handeln nichtstaatlicher Akteure zurückzuführen (Burley/Mattli 1993: 43; Mattli/Slaughter 1998: 180-181; Stone Sweet/Sandholtz 1997: 305). Die entscheidenden Triebkräfte der Konstitutionalisierung seien demnach Gerichte unterhalb der nationalen Verfassungsebene und exportorientierte Unternehmen gewesen. Deren politische Orientierungen und Präferenzen hätten sich infolge der anfänglichen wirtschaftlichen Integration schrittweise auf die supranationale Ebene verschoben: Während sich erstere mehr Autonomie von den höheren Gerichtsinstanzen ihrer nationalen Rechtssysteme erhofften, verlangten letztere eine Ausweitung und Absicherung ihrer Rechte in der auf dem Weg zu einem Gemeinsamen Markt befindlichen Wirtschaftsordnung. Durch ihre Klage- und Urteilspraxis ermächtigten sie den Europäischen Gerichtshof $(\mathrm{EuGH}) \mathrm{zu}$ einer ständigen Rechtsprechung, die inkrementell Normen einer supranationalen Rechtsordnung verankerte. Intergouvernementalistische Ansätze sehen das Design dieser Rechtsordnung im Wesentlichen als Funktion nationaler Interessen der Mitgliedsstaaten bzw. dominanter Interessenkoalitionen (Garrett 1992: 534-535; Phelan 2018: 1566-1567). Die EU-Verträge seien demnach unter Bedingungen von incomplete contracting zustande gekommen, was ihre Anwendung und die Überprüfung ihrer Einhaltung kostspielig machte. Um hohe wirtschaftliche Interdependenz in dauerhafte Kooperationsgewinne umzumünzen, schufen die Mitgliedsstaaten daher ein supranationales System an Überwachungs- und Schlichtungsmechanismen. Die Anrufung des EuGH durch nichtstaatliche Akteure mag in Einzelfällen staatliche Autorität infrage stellen - auf lange Sicht stärkt sie aber den Binnenmarkt und damit das zentrale Interesse der Mitgliedsstaaten. Beide klassischen Ansätze führen die progressive Verrechtlichung der EU also kausal auf die Übersetzung funktionaler Nachfrage in institutionelle Entscheidungen mittels bestimmter politischer Prozesse - spillover vs. zwischenstaatliche Verhandlungen - zurück.

Die Vergleichende Regionalismusforschung hat unter Verweis auf empirische Befunde aus anderen Weltregionen argumentiert, dass diese Integrationslogik außerhalb Europas oft nicht greift. Selbst wo eine relativ starke wirtschaftliche Interdependenz vorliegt, übersetzt diese sich nicht notwendigerweise via funktionalem spillover in eine zunehmende Integration anderer Politikfelder (vgl. bereits Grieco 1997: 171-173). Dies lässt sich etwa in Ostasien beobachten, einer Region, die nicht nur starke zwischenstaatliche Handelsbeziehungen, sondern auch ausgedehnte transnationale Wirtschaftsnetzwerke aufweist. Entweder hat sich aus diesen Interdependenzen keine Nachfrage nach supranationalen Organisationen ergeben, oder die postulierten Transmissionsmechanismen blieben in diesen Fällen wirkungslos (Jetschke/Katada 2016: 232-233). Umgekehrt dominiert in Teilen Afrikas ein staatlich getriebener und von regime boosting (Söderbaum 2004: 425-428) motivierter Top-Down-Regionalismus, dessen zumindest formell sehr ausgeprägte organisatorische Komponente disproportional $\mathrm{zu}$ den relativ geringen gesellschaftlichen und 
wirtschaftlichen Interdependenzen erscheint (Hartmann 2016: 272). Amitav Acharya (2016: 117) bringt die Kritik auf den Punkt:

\begin{abstract}
"Neo-functionalism [...] cannot tell us why regionalisms in Asia, Latin America, and Africa developed with fundamentally different goals (keeping, rather than bypassing, sovereignty) in the first place, and in a strikingly different manner (with much less institutionalization) from the EU. Nor could it capture the differences in goals and methods among the various regionalisms in the developing world. Local conditions, determinants, and process dynamics matter [...].“
\end{abstract}

Folgerichtig kommen klassische Theorien zur Erklärung von außereuropäischen Regionalisierungsprozessen oder Integrationsunterschieden zwischen Regionen nur selten zum Einsatz (vgl. Schimmelfennig 2018). Das gilt auch für das Feld der rechtlichen Integration. Während einige AutorInnen etwa die Designs verschiedener regionaler Streitbeilegungsmechanismen mit aus divergierender wirtschaftlicher Interdependenz entspringenden Funktionslogiken erklären (Kanthak 2012: 81; Krapohl et al. 2010: 173-175), verweisen andere auf Fälle wie den der ASEAN, in denen derartige Argumente nicht oder nur bedingt greifen (Korte 2012: 101; Solingen 2008: 288-289). Stattdessen ziehen vergleichend orientierte Ansätze meist machtpolitische Faktoren wie den Einfluss hegemonialer Akteure (Grieco 1997; Katzenstein 2005; Mattli 1999), die Präferenzen innerstaatlicher Akteurskonstellationen und Eliten (Acharya 2016; Söderbaum 2004; Solingen 2008), regionale Identitäten (Checkel 2016; Hemmer/Katzenstein 2002) und kognitive Prädispositionen (Acharya 2009) zur Erklärung regionaler Unterschiede und Eigenheiten heran.

Die Vergleichende Regionalismusforschung hat durch diese Arbeiten die Grenzen der klassischen Integrationstheorien aufgezeigt und innovative Erklärungen für Integrationsunterschiede entwickelt - allerdings um den Preis, dass sie die Differenzen zum Teil typologisch überzeichnet. So wird häufig ein europäisch-legalistischer mit einem (südost-)asiatisch-informellen Ansatz kontrastiert (Higgott 2014; Katzenstein 2005). In diesem Zusammenhang räumen die meisten AutorInnen strukturell-exogenen Erklärungsfaktoren wie ökonomischen Determinanten, Interessen, politischen Systemen und Kulturen oder Normkonfigurationen Vorrang ein, wogegen die agency regionaler Akteure in den Hintergrund rückt. ${ }^{4}$ Damit replizieren sie letztendlich das Erklärungsmuster der klassischen Theorien, welche die oft hinter den Erwartungen zurückbleibenden Integrationsfortschritte außerhalb Europas auf strukturell-objektive Kontextbedingungen - z.B. sozioökonomische und politisch-ideologische Konstellationen in und zwischen den jeweiligen Staaten - zurückführten (Haas 1961: 377-378). Zum Zwecke kausaler Theoriebildung mag eine strukturalistische Perspektive in manchen Fällen sinnvoll sein, jedoch leistet sie mitunter einem holzschnittartigen Determinismus Vorschub, der die vielfältigen Nuancen, Ambiguitäten und Kontingenzen regionaler Integrationsprozesse insbesondere in ihrer historischen Entwicklung ausblendet (vgl. kritisch Spandler 2018a: 192).

4 Die Forschung zur interregionalen Diffusion von policies und institutionellen Designs zwischen Regionen (z.B. Börzel/Risse 2012; Jetschke 2017) ist von dieser Kritik auszunehmen. 
Die Emanzipation der Vergleichenden Regionalismusforschung von den dominanten Integrationstheorien hat einen extrem wichtigen Beitrag zur Entwicklung einer globalen IB-Forschung geleistet. Eine prinzipielle Zurückweisung konventioneller Konzepte und Modelle zum Zwecke der eigenen Profilbildung ist dabei jedoch letztendlich kontraproduktiv (Warleigh-Lack 2006: 756-758). Mir erscheint es lohnender, die Auseinandersetzung zur Theorieentwicklung zu nutzen. Erstens können vergleichende Studien die bisweilen abstrakt-theoretische Diskussion über die Vergleichbarkeit der EU empirisch substantiieren (Murau/Spandler 2016: 940-941). Zweitens ermöglichen solche Studien Rückschlüsse über die scope conditions einzelner Ansätze oder Hypothesen (Acharya 2016: 125-126). Schließlich kann diese Auslotung von Gültigkeitsgrenzen bestehender Ansätze auch Katalysator für theoretische Innovationen sein - entweder durch die Modifikation etablierter Konzepte und Mechanismen, oder durch die Infragestellung ihrer ontologischen und epistemologischen Grundannahmen. Die im folgenden Abschnitt entwickelte Idee der normativ-diskursiven Verfasstheit regionaler Integration ist ganz in diesem Sinne von der Kritik an den klassischen Narrativen der EU-Integrationstheorie animiert.

\section{Normative Diskurse - ein Framework}

Grundlage des in diesem Beitrag verfolgten Ansatzes ist die Annahme, dass die institutionelle Entwicklung regionaler Organisationen durch normative Diskurse getrieben ist. Mit diesem Konzept schlägt der Artikel eine Brücke zwischen Theorien des kommunikativen Handelns in den IB (Müller 1994; Risse-Kappen 1995; Schimmelfennig 1997) und institutionalistischen Konzepten der English School (Buzan 2004). Der Begriff umfasst im konkreten Zusammenhang die Prozesse, in denen regionale Akteure die institutionellen Formen ihrer Beziehungen unter Berufung auf internationale Prinzipien und Normen aushandeln. Damit betont er in Abgrenzung zur utilitaristischen Rationalität der klassischen Integrationstheorien - in der sich institutionelle Equilibrien aus den Kosten-Nutzen-Kalkulationen ergeben, die individuelle AkteurInnen auf der Grundlage exogener Präferenzen anstellen die argumentative Legitimierung und Delegitimierung als irreduziblen Bestandteil jedes regionalen institution buildings.

Im Gegensatz zum Angelpunkt der Debatte in der Zeitschrift für Internationale Beziehungen (zib) in den 1990er-Jahren ist die hier vertretene Idee des normativen Diskurses agnostisch in Bezug auf die Handlungslogik der beteiligten AkteurInnen (vgl. Krebs/Jackson 2007: 40). Sie folgt damit empirischen Befunden und theoretischen Weiterentwicklungen nach der Jahrtausendwende, wonach eine klare Trennung zweckrationaler und verständigungsorientierter Kommunikationssituationen schwierig, wenn nicht gar unmöglich ist (Deitelhoff/Müller 2005: 170-171). Stattdessen nimmt sie an, dass normative Diskurse sowohl instrumentell-rhetorische Überlegungen der beteiligten AkteurInnen als auch Elemente eines dialektischen truth seeking (Risse 2000: 6-7) integrieren. Auch wenn AkteurInnen vorrangig strategisch kommunizieren, müssen sie ihre Argumente durch Begründungen legitimie- 
ren, was letztendlich - wenn auch oft nur implizit - durch normative Bezüge erfolgt (vgl. Holzinger 2001: 269).

Die Integrationsforschung hat sich bereits in den späten 1990er-Jahren mit kommunikativen Ansätzen verschiedener Couleur auseinandergesetzt (Diez 1996; 1999; Jachtenfuchs et al. 1998; Meyer 1999; Risse-Kappen 1996) und diese auch zur Analyse und normativen Bewertung der rechtlichen Dimension der EU angewendet (Joerges/Neyer 1997; Wiener 1998). Trotz der großen Differenzen zwischen einzelnen AutorInnen hat sich daraus ein breites Forschungsfeld entwickelt, das den kommunikativ vermittelten Zusammenhang zwischen institutionellen Regionalisierungsprozessen und Gemeinschaftsvorstellungen ins Zentrum rückt. Luk Van Langenhove (2011: 75-78) konzipiert beispielsweise integration speak als Triebkraft für zunehmende Institutionalisierung auf regionaler Ebene. In ihrer postfunktionalistischen Integrationstheorie argumentieren Liesbet Hooghe und Gary Marks (2009: 23) umgekehrt, dass öffentliche Debatten in den EU-Mitgliedsstaaten auch nationale Identitäten mobilisieren und dadurch funktionalistische Integrationsdynamiken auf europäischer Ebene aushebeln können.

Während die Rolle solcher Diskurse als Vermittler zwischen Identitäten und institutionellen Formen weitgehend anerkannt ist, wird ihre normative Dimension jedoch oft stiefmütterlich behandelt. Dabei hat gerade die kommunikative Handlungstheorie die Bedeutung eines gemeinsamen lebensweltlichen Bewertungsrahmens als Voraussetzung für diskursive Verständigung zwischen den beteiligten AkteurInnen betont (Müller 1994: 26-27). Zwar haben Studien des strategischen bzw. rhetorischen Handelns von EU-Akteuren die Berufung auf gemeinsame europäische Normen und Wertvorstellungen als wichtige Legitimationsressource identifiziert (Rittberger/Schimmelfennig 2006; Schimmelfennig 2003: 541-542). Insofern dienen sie als maßgebliche Referenzen für das hier entworfene Framework. Aufgrund der Beschränkung auf den europäischen Raum haben sie die Genese und Dynamik dieses Rahmens jedoch nie systematisch problematisiert. Vielmehr wurden sicherlich vor dem Hintergrund des spezifischen Forschungsinteresses zurecht - die fundamentalen Werte der liberaldemokratischen Staatengemeinschaft als konstitutiv für die strategischen EU-Integrationsdiskurse vorausgesetzt (Schimmelfennig 2002: 599-600; vgl. kritisch Seidendorf 2006: 101-103). In komparativer Perspektive wird die Frage nach dem normativen Referenzrahmen von Integrationsdiskursen jedoch unweigerlich zu einer empirischen (Spandler 2018b: 186), ist es doch ein Gemeinplatz sowohl der Vergleichenden Regionalismusforschung als auch der Area Studies, dass Regionalorganisationen jenseits des europäischen Raumes von spezifischen Kooperationsprinzipien - z.B. dem sogenannten ASEAN Way - geprägt sind.

Mit dem Konzept der Primärinstitutionen stellt die English School ein begriffliches Instrumentarium zur Verfügung, das im Gegensatz zur Idee einer liberalen Gemeinschaft die nötige analytische Offenheit für temporal und regional variierende Normen bietet und eine vergleichende Perspektive ermöglicht, die die europäische Erfahrung transzendiert, aber nicht ausschließt. Primärinstitutionen sind grundlegende Zusammenhänge von Normen und Praktiken internationaler Beziehungen 
(Buzan 2004: 167-176). Ihr fundamentaler, tiefenstruktureller Charakter unterscheidet sie von den konkreten Regelzusammenhängen, die in der English School als Sekundärinstitutionen gelten und die im neo-liberalen Institutionalismus als eigentliche Kernbestandteile regionaler Organisationen angesehen werden. Hedley Bull (1977: 101-229) hat in seiner bis heute maßgeblichen Analyse der modernen internationalen Gesellschaft fünf solcher Institutionen identifiziert - Diplomatie, Völkerrecht, Kriegsführung, das Mächtegleichgewicht und die besonderen Rechte und Pflichten von Großmächten. Daneben haben sich spätestens seit Mitte des 20. Jahrhunderts aber auch regionale Institutionen sowie regional spezifische Interpretationen globaler Institutionen entwickelt. Solche Prozesse sind wahlweise als Vertiefung oder Fortentwicklung der rudimentären globalen Staatengesellschaft, als Lokalisierung oder als Gegenentwurf zu hegemonialen Ordnungsvorstellungen gedeutet worden (Buzan 2009: 35-39; Diez et al. 2011: 118-119; Zhang 2015: 365-367).

Primärinstitutionen bringen grundlegende Vorstellungen über die konstitutiven Akteure, Spielregeln und Rationalitäten einer internationalen Ordnung zum Ausdruck. Sie stellen damit den zentralen Referenzrahmen - gewissermaßen die institutionalisierte Lebenswelt im Habermasschen Sinne oder die diskursive Opportunitätsstruktur (Koopmans/Olzak 2004: 202-203) - für die normative Rückbindung von Integrationsdiskursen dar. Die legitimierende Kraft konkreter Argumente in regionalen Integrationsdiskursen hängt davon ab, ob sie mittels framing implizite oder explizite Verbindungen zu Primärinstitutionen herstellen und dadurch Resonanz und diskursive Macht erlangen können. Frames sind Zusammenhänge von Aussagen, die der Interpretation und Legitimation sozialer Sachverhalte und Handlungen dienen. In Integrationsdiskursen begründen sie spezifische Positionen bezüglich der institutionellen Ausgestaltung regionaler Ordnung.

In Gegensatz zu klassischen Integrationstheorien, für die der normative Kontext von Integration entweder irrelevant oder exogen gegeben ist, hebt dieser Ansatz hervor, dass selbst scheinbar wertneutrale Argumente für Integration auf einem normativen Rahmen beruhen, dessen Gültigkeit stets Gegenstand diskursiver Aushandlungen werden kann. Schon Ernst B. Haas (1975: 8, 86-91) wies darauf hin, dass die Erklärungskraft funktionalistischer Ansätze durch Wandel in den Werten, Motiven und Rationalitäten von EntscheidungsträgerInnen stark eingeschränkt werden könne. Neofunktionalistische Ansätze versuchen, dieser Kontingenzen innerhalb ihrer rationalistischen Ontologie Herr zu werden, indem sie auf gleichsam bremsende Ausgleichskräfte wie z.B. innerstaatliche Einschränkungen autonomen Regierungshandelns oder ein negatives Integrationsklima eingehen (Niemann 2006: 47-50). Dabei nehmen sie jedoch weiterhin an, dass funktionalistische Kräfte als objektive und unabhängige Kausalmechanismen integrationsfördernd wirken, ohne dass sie dafür in konkreten Auseinandersetzungen über institutionellen Wandel mobilisiert werden müssten. Der hier präsentierte Ansatz nimmt einen anderen Weg: Er endogenisiert Funktionalismus als frame, dessen sich politische Akteure zur Legitimierung von institutionellem Wandel bedienen können, aber nicht müssen. Die legitimierende Kraft dieses frames beruht auf einer Rationalität, die regionale Organisationen an ihrer nachfrageorientierten Problemlösungskapazität und der Bereit- 
stellung von Gemeinschaftsgütern für die Bevölkerungen der Mitgliedsstaaten misst. Marktliberale und liberaldemokratische Prinzipien sind intuitive Kandidaten für Primärinstitutionen, die eine derartige Rationalität konstitutiv stützen.

Die Primärinstitutionen einer Region definieren also einen Möglichkeitsraum für Stabilität und Wandel von Regionalorganisationen. Dieser bleibt jedoch stets ambig und kann dementsprechend keine deterministische Kausalität entfalten. Denn aus unterschiedlichen Primärinstitutionen lassen sich teils widersprüchliche Handlungsanweisungen ableiten - man denke etwa an die Spannungen zwischen menschenrechtlichen und Souveränitätsansprüchen, wie sie in den Debatten um humanitäre Interventionen zum Ausdruck kommen (Wheeler 2000: 21-52). Zudem ist die Umsetzung in konkrete Sekundärinstitutionen stets unterdeterminiert - prinzipiell lässt sich durch ein abstraktes Set an Prinzipien und Normen stets eine Vielzahl von Praktiken legitimieren (Knudsen 2018: 38-39). Zuletzt ist die Gültigkeit einzelner Primärinstitutionen häufig umstritten. Gerade die diskursive Auseinandersetzung über die normativen Grundlagen von Kooperation ermöglicht zugleich deren Transformation, wie schon in der zib-Debatte betont wurde (z.B. Schimmelfennig 1997: 224). Neue Primärinstitutionen können konstruiert, existierende reinterpretiert werden oder ihre normative Kraft verlieren. Normative Diskurse fungieren damit in beide Richtungen als Vermittlungspraktiken zwischen Primär- und Sekundärinstitutionen. Zum einen bewirken sie eine Entwicklung regionaler Organisationen in Kompatibilität mit den normativen Grundsätzen regionaler Ordnung. Gleichzeitig können sie diesen Referenzrahmen auch zugunsten bestimmter politischer Präferenzen verschieben. Langfristig folgt regionale Integration daher dem Muster einer pfadabhängigen Koevolution von Primär- und Sekundärinstitutionen (vgl. Spandler 2015: 609-621). Welche Handlungslogik aber diesen Wandel treibt, lässt sich aus theoretischen Überlegungen oder strukturellen Bedingungen nicht a priori ableiten, sondern muss - und hier zeigt sich eine Nähe zu praxistheoretischen Ansätzen (Pouliot 2007; 2008; Kornprobst 2011) - anhand der diskursiven Praktiken der beteiligten AkteurInnen rekonstruiert werden.

Auf Grundlage dieser Überlegungen untersucht der folgende Abschnitt die normativen Begleitdiskurse zentraler rechtlicher Integrationsprozesse in der EU und der ASEAN. Das Untersuchungsdesign folgt der Idee einer interpretativen vergleichenden Fallstudie, die die komparative Methodik weniger rigide interpretiert als streng kausal orientierte Ansätze. Vergleichende Fallstudien verbinden die thick description interpretativer Ansätze mit der Möglichkeit, aufgrund einer strukturierten und fokussierten Herangehensweise (George/Bennett 2005: 67-72) fallübergreifende Muster oder Unterschiede zu identifizieren. Sie ermöglichen so die Interpretation und Erklärung vergleichbarer historischer Prozesse in einer Weise, die kontextsensitive, begrenzte Generalisierungen und theoretische Rückschlüsse über zugrundeliegende Mechanismen zulässt (Ragin 1987: 35). Dementsprechend werden beide Fälle durch die Linse des hier präsentierten Frameworks untersucht, aus dem sich Leitfragen für die Analyse der jeweiligen Diskurse ableiten lassen. So interpretiert die Analyse die zentralen frames der Diskurse auf implizite oder explizite Beziehungen zu den Primärinstitutionen der jeweiligen Region. Ziel ist weder eine um- 
fassende historiographische Rekonstruktion der jeweiligen Integrationsprozesse, noch die Isolation von Variablen und Korrelationen. Vielmehr soll im Sinne einer Plausibilitätsprüfung das theoretische Argument empirisch untermauert werden, dass (a) regionale Integrationsprozesse diskursiv verfasst sind, und (b) die Resonanz spezifischer Argumentationsmuster in diesen Diskursen vom normativen Kontext regionaler Primärinstitutionen bedingt wird. Die Kontrastierung der primärinstitutionellen Kontexte Europas und Südostasiens dient also nicht einer verallgemeinerbaren kausalen Erklärung unterschiedlicher Integrationsgrade, sondern verleiht der Interpretation funktionalistischer Integrationslogik als Diskursmotiv Stichhaltigkeit. Dennoch lassen sich aus ihr durchaus auch begrenzte Generalisierungen über die Wirkung bestimmter struktureller Eigenschaften des primärinstitutionellen Kontexts auf die Dynamik regionaler Diskurse ableiten - insbesondere hinsichtlich der Resonanzbedingungen für funktionalistische frames.

\section{Verrechtlichungsprozesse in der EU und $A S E A N^{5}$}

Die folgende Analyse konzentriert sich auf jeweils eine Kernphase in der Entwicklung der Rechtsordnung beider Organisationen. Der 1992 unterzeichnete Vertrag von Maastricht, durch den die Europäischen Gemeinschaften (EG) in die Europäische Union überführt wurden, war ein Meilenstein in der Konstitutionalisierung einer europäischen Rechtsordnung, insbesondere durch die Schaffung einer Unionsbürgerschaft für alle StaatsbürgerInnen der EU-Mitgliedsstaaten. Die ASEANStaaten unterzeichneten 2007 eine Charta, in der existierende rechtliche Mechanismen, insbesondere regionale Streitbeilegungsverfahren, formalisiert und reformiert wurden und durch die die Organisation eine Rechtspersönlichkeit erhielt. Ganz im Sinne des interpretativ-vergleichenden Designs zielt diese Fallspezifizierung nicht auf eine größtmögliche Parallelisierung im Hinblick auf den Politikbereich ab, wie sie etwa durch die Betrachtung von Streitbeilegungsmechanismen in beiden Organisationen möglich gewesen wäre. Sie berücksichtigt stattdessen auch Kriterien wie den Politisierungsgrad und die archivarische Zugänglichkeit der historischen Debatten, um das Illustrationspotential des Vergleichs zu maximieren.

Trotz der offensichtlichen Unterschiede in Chronologie, Ausmaß und Charakter weisen beide Prozesse jedoch auch Gemeinsamkeiten auf. Zum einen wurden in beiden Fällen klassische Vorstellungen des positiven Völkerrechts als zwischenstaatliche Angelegenheit infrage gestellt. Darüber hinaus, und das zeigt die Analyse, erschließt sich ihre spezifische Entfaltung nicht allein aus den Präferenzkonstellationen der beteiligten AkteurInnen in den unmittelbar vorangehenden Verhandlungsprozessen, also der Regierungskonferenz der EU-Mitgliedsstaaten einerseits und dem Drafting-Prozess der ASEAN andererseits. Sie stellen auch keine optimalen Lösungen für transnationale governance-Probleme dar. Vielmehr sind sie als

5 Die Ausführungen in diesem Abschnitt stützen sich auf weiterführende Analysen in Spandler (2018a, 99-138). 
(vorläufige) Endpunkte langjähriger normativer Diskurse zu verstehen, die die Grundlagen für die institutionelle Ausgestaltung legaler Integration schufen. ${ }^{6}$

\subsection{Die Konstitutionalisierung des EG-Rechts und die Unionsbürgerschaft}

Der zweite Teil des Maastrichter Vertrages formalisierte den Status des Unionsbürgers für alle BürgerInnen der EU-Mitgliedsstaaten und gewährte ihnen Freizügigkeits- und Aufenthaltsrechte, das Wahlrecht bei Kommunalwahlen im Wohnsitzland, diplomatischen Schutz außerhalb der EU-Grenzen sowie das Petitionsrecht beim Europäischen Parlament (EP). Darüber hinaus schuf der Vertrag die Position einer Ombudsperson, die als Interessenvertreterin der UnionsbürgerInnen auf Gemeinschaftsebene fungiert. Einige Bestimmungen über die Freizügigkeit und das Aufenthaltsrecht waren zwar bereits in den Gründungsverträgen der EG enthalten, dort jedoch auf ArbeitnehmerInnen beschränkt. Insofern war der Maastrichter Vertrag ein Meilenstein der rechtlichen Integration. Die Unionsbürgerschaft konstituierte subnationale AkteurInnen als legitime Subjekte regionaler Politik und trug dadurch maßgeblich zur Emergenz eines regionalen Rechtsgefüges bei, das die etablierten zwischenstaatlichen Strukturen nationaler Verfassungsordnungen und des Völkerrechts ergänzte.

Frühe Figurationen eines Bürgerschaftsdiskurses ${ }^{7}$ finden sich bereits wenige Jahre nach der Gründung der EG. So interpretierte der damalige Sozialkommissar Lionello Levi Sandri bereits 1961 vor der Parlamentarischen Versammlung die Freizügigkeit der GemeinschaftsbürgerInnen als „erstes Element einer Europäischen Bürgerschaft" (Levi Sandri 1961: 135, meine Übersetzung). Auf der Ebene der Staatsund Regierungschefs der Mitgliedsstaaten fand diese Idee jedoch zunächst nur wenig Resonanz. Zwar schlug der damalige italienische Premierminister Giulio Andreotti 1972 eine Europäische Bürgerschaft als Ergänzung zur Staatsbürgerschaft in den Mitgliedsländern vor, doch die daran anschließenden Initiativen und Arbeitsgruppen zur Einführung von Sonderrechten und einer Passunion blieben vorerst fruchtlos (Wiener 1998: 74). Noch 1979 musste der Vorsitzende des Rechtsausschusses des EP, Alfons Bayerl, bei der Vorstellung eines Berichts zur Bürgerschaft SkeptikerInnen aus anderen EG-Organen und den Mitgliedsstaaten beschwichtigen:

„Nobody wanted a ,superconstitution“, and no Member of the European Parliament who supported the idea of drawing up rights for the Community's citizen wanted to take from national authorities the powers which devolved on them in the field of fundamental rights“" (European Parliament 1979: 44).

Ein Grund für diese ambivalente Entwicklung liegt in der Opportunitätsstruktur dieser normativen Diskurse. Drei Primärinstitutionen waren konstitutiv für die an-

6 Die Analyse schließt damit auch an die konstruktivistische Völkerrechtsforschung an, die auf die kommunikative Konstitution von Verrechtlichungsprozessen aufmerksam macht (z.B. Deitelhoff 2009; Finnemore/Toope 2001; Wiener 1998).

7 Die Analyse begreift Bürgerschaft im Sinne eines rechtlichen Status, der die Mitgliedschaft in und Partizipation an einer politischen Gemeinschaft begründet. 
fänglichen Auseinandersetzungen zur Bürgerschaft. Erstens hatte die politische Elite der EG-Mitgliedsstaaten sich nach dem Ende des Zweiten Weltkriegs unter Berufung auf wirtschaftliche und sicherheitspolitische Überlegungen weitgehend vom imperialen Souveränitätsbegriff der Kolonialzeit einerseits und der Westfälischen Idee souveräner Gleichheit andererseits zumindest in ihren jeweiligen Reinformen verabschiedet. An deren statt hatte die Vorstellung von Europa als Raum gebündelter Souveränität (pooled sovereignty) schrittweise den Status einer Primärinstitution erlangt, die vor allem mit der Idee eines Gemeinsamen Marktes verknüpft war (Diez et al. 2011: 126-127). Die damit verbundene Verschiebung politischer Autorität auf die regionale Ebene blieb allerdings selbst im wirtschaftlichen Bereich unvollständig. Denn als zweite zentrale Primärinstitution wurde der Nationalismus als Bezugspunkt für die Entwicklung des politischen Rahmens der EG bestärkt, wie sich unter anderem in der Rhetorik des Europe des patries und dem französischen Boykott des Ministerrats durch die Politik des leeren Stuhls zeigte (Milward 1992: 2-3 und passim). Gemäß dem Prinzip der nationalen Selbstbestimmung konnten Einzelpersonen InhaberInnen von Rechten gegenüber den nationalen Staatsapparaten, nicht aber gegenüber transnationalen Organisationen sein. Schließlich blieb drittens die europäische Rechtslehre auch nach der Gründung der EG zunächst einer positiven Interpretation der Primärinstitution des Völkerrechts verpflichtet. Demzufolge waren Staaten die einzigen Quellen einer überstaatlichen Rechtsordnung und ausschließlich solchen Regeln verpflichtet, die sie selbst aus freien Stücken eingingen. Die Gründung des EuGH war mit dieser Doktrin durchaus vereinbar, entsprach sie doch der gängigen völkerrechtlichen Praxis, Rechtsprechung durch zwischenstaatliche Übereinkunft an internationale Gerichte zu übertragen. Liberaldemokratische Prinzipien waren dagegen zunächst nicht auf regionaler Ebene institutionalisiert (Thomas 2017: 226-227). Der rechtliche Status von Einzelpersonen in der EG konnte in dieser Auffassung nicht über ihre Rechte und Pflichten als BürgerInnen eines Mitgliedsstaates hinausgehen.

Diese primärinstitutionellen Bedingungen schwächten einerseits die Stellung von BürgerschaftsbefürworterInnen. Gleichzeitig katalysierten sie jedoch zwei primärinstitutionelle Dynamiken, die den konstitutiven Kontext der Debatten langfristig zugunsten integrationsfreundlicher Positionen transformierten. Erstens etablierte der EuGH durch seine ständige Rechtsprechung in den 1960er- und 1970er-Jahren schrittweise die Prinzipien der unmittelbaren Anwendbarkeit und des Vorrangs des Gemeinschaftsrechts. Die in reziproker Sicht vor allem von neofunktionalistischen Ansätzen hervorgehobene Bedeutung des Gerichtshofes für den Einigungsprozess verschleiert mitunter die Tatsache, dass ihm diese Funktion nicht von Beginn an eingeschrieben war. Ganz im Gegenteil: Die Mitgliedsstaaten hatten ihn ursprünglich als Bollwerk gegen eine zu expansive Kompetenzaneignung der Kommission geschaffen (Alter 2009: 123-125). So führt Kapitel IV des Gründungsvertrags der Europäischen Gemeinschaft für Kohle und Stahl von 1951, das den Zuständigkeitsbereich des Gerichtshofs definiert, beinahe ausschließlich potenzielle Vertragsverletzungen der Hohen Behörde auf - also des Vorgängers der Kommission. Erst nach und nach definierte der Gerichtshof seine Rolle als Integrationsmotor. Seine schritt- 
weise Emanzipation legitimierte er durch innovative normative Argumente. Er berief sich insbesondere auf die Widersprüche, die sich aus der Koexistenz des klassischen Völkerrechts und der Primärinstitution der pooled sovereignty ergaben. Wenn zwischenstaatliche Verträge supranationale Entscheidungsorgane mit Einfluss auf das Leben der europäischen BürgerInnen schaffen, dann muss die so entstandene Rechtsordnung individuelle Rechte gegenüber diesem neuen Souverän garantieren - so das Urteil im wegweisenden Fall Van Gend \& Loos. Rechtswissenschaftliche Analysen haben darauf hingewiesen, dass die eigentliche Bedeutung dieser Urteile nicht in der unmittelbaren Auslegung konkreter Gesetze lag, sondern in der Entwicklung einer normativen Doktrin, die die Gültigkeit klassischer Völkerrechtskonzeptionen einschränkte (Alter 2009: 130-135). So schuf der Gerichtshof die Grundlage für eine gemeinschaftliche Rechtsordnung als neue regionale Primärinstitution. Die Entwicklung schwächte die Autorität nationaler Regierungen, da Fragen, die zuvor gemäß dem völkerrechtlichen modus operandi in intergouvernementalen diplomatischen Settings verhandelt wurden, nun einer rechtlichen Diskurslogik unterworfen wurden.

In einem zweiten transformativen Prozess formulierten einzelne Gemeinschaftsorgane liberaldemokratische Prinzipien als Grundelement des EG-Wertekanons (Diez et al. 2011: 130-132; Thomas 2017: 228-235). Der Birkelbach-Bericht der Parlamentarischen Versammlung von 1961 und der Luxemburger Bericht, der 1970 von einem ExpertInnenausschuss erstellt und später vom Rat angenommen wurde, wurden zu wichtigen Bezugspunkten in dieser Hinsicht. Auf dem Kopenhagener Gipfel 1973 einigten sich die Staats- und Regierungschefs auf die Erklärung zur europäischen Identität, in der die Demokratie ebenfalls eine zentrale Rolle spielte. Mit einigen Einschränkungen waren die gemeinschaftliche Rechtsordnung und Demokratie Ende der 1970er-Jahre so als neue europäische Primärinstitutionen etabliert.

BefürworterInnen einer europäischen Bürgerschaft machten sich diese Entwicklungen in ihrem framing zunutze. Sie beriefen sich auf die beiden neuen Primärinstitutionen, um das Individuum als InhaberIn legitimer Rechtsansprüche gegenüber der Gemeinschaft zu konstruieren (Haltern 2004: 180). Dies stärkte auch die Position des EP, das sich als Repräsentant individueller Rechte verstand. So verband etwa der nach seinem Vorsitzenden auch Bayerl Report genannte Bericht des Rechtsausschusses von 1979 die Idee einer Gemeinschaftsbürgerschaft mit der Forderung nach einer Demokratisierung der EG: „Only equal treatment of citizens in every member state can guarantee in the long term a democratization of the Community and contribute to its further development" (Legal Affairs Committee 1979). Der unter Federführung von Altiero Spinelli im Februar 1984 veröffentlichte Vertragsentwurf zur Gründung der Europäischen Union bekräftigte die liberaldemokratischen Grundsätze der Gemeinschaft sowie die Prinzipien der unmittelbaren Anwendbarkeit und des Vorrangs des EG-Rechts und verband diese Ideen mit Regeln für eine Unionsbürgerschaft. Der vorgeschlagene Rahmen trug freilich auch den nationalen Rechtstraditionen Rechnung, indem er die Unionsbürgerschaft von der Staatsbürgerschaft in einem EU-Mitgliedsstaat abhängig machte und individuelle Rechte aus 
den „gemeinsamen Grundsätzen der Verfassungen der Mitgliedsstaaten und der Europäischen Konvention zum Schutz der Menschenrechte und Grundfreiheiten“ ableitete (Europäisches Parlament 1984, Art. 4.1).

Mit seiner Initiative verlieh das EP dem Bürgerschaftsdiskurs eine neue Dynamik (Maas 2007: 35). Der Europäische Rat beschloss nur wenige Monate nach der Veröffentlichung des Vertragsentwurfs die Einsetzung einer Arbeitsgruppe zur Förderung einer gemeinschaftlichen Identität unter den BürgerInnen der EG. Der zu diesem Zweck gegründete Adonnino-Ausschuss legte dieses Mandat recht weit aus. In seinen zwei Berichten schlug er Regeln für eine Gemeinschaftsbürgerschaft vor, die weitgehend die des Maastrichter Vertrags vorwegnahmen. Der Ausschuss begründete diese Vorschläge einerseits mit Argumenten, die die Marktlogik der Primärinstitution pooled sovereignty widerspiegelten. So bezeichnete er beispielsweise die Freizügigkeit als ,notwendige Ergänzung zu dem Programm zur Vervollkommnung des Binnenmarktes, dem mit Recht oberste Priorität zuerkannt worden ist" (Adhoc-Ausschuss 1985: 128). Andererseits beschreibt er Bürgerrechte als Mittel zur Verteidigung der Prinzipien der EG und zur Stärkung ihrer demokratischen Legitimität und Glaubwürdigkeit.

Das Schengen-Abkommen von 1985 und die Einheitliche Europäische Akte von 1986 spiegelten diese Ideen nur sehr bedingt wider. Der Vorschlag der dänischen Regierung, das Kommunalwahlrecht im Wohnsitzland in die Akte aufzunehmen, fand keine Mehrheit, und die Regelung der Freizügigkeit blieb fest in der Hand der Mitgliedsstaaten (Maas 2007: 38). Die Europäische Kommission kritisierte den Rat dementsprechend und forderte unter Verweis auf die Primärinstitutionen der Demokratie und der gemeinschaftlichen Rechtsordnung weiterreichende Maßnahmen:

„Die künftige Gemeinschaftsarbeit wird eine wesentlich unmittelbarere Auswirkung auf alle Bürger haben als in der Vergangenheit. Der Bürger muss sich dessen bewußt sein. Dieses Bewußtsein [...] ist [...] die Voraussetzung dafür, daß der europäische Bürger seine politischen Erwartungen auf Gemeinschaftsebene formuliert, wie er dies auf nationaler, regionaler oder lokaler Ebene gewohnt ist" (Europäische Kommission 1988: 2).

„Nach dreißig Jahren europäischer Integration hat die kontinuierliche und manchmal spektakuläre Entwicklung des Gemeinschaftsrechts zu einer Rechtsordnung geführt, die alle gesellschaftlichen Bereiche erfasst: Sowohl die Wirtschaftsakteure als auch der europäische Bürger im Alltagsleben sind davon betroffen. Das Gemeinschaftsrecht [...] gibt ihnen besondere Rechte, auf die sie sich berufen können. Allerdings muß der Bürger diese Rechte in seinem alltäglichen und beruflichen Leben kennen. Das Stimmrecht bei Kommunalwahlen wäre hierbei der beste Beweis dafür, daß es tatsächlich ein Europa der Bürger gibt. [...] Dieses Recht [...] fördert die demokratische Teilhabe des Bürgers am Gemeindeleben seines Wohnorts" (Europäische Kommission 1988: 26-30).

Derart unter Druck gesetzt beschlossen die Staats- und Regierungschefs auf ihrem Gipfeltreffen 1990 in Rom, die Definierung einer europäischen Bürgerschaft auf die Agenda der Regierungskonferenz zur Politischen Union von 1990/91 zu setzen (Europäischer Rat 1990). Die Verhandlungspositionen der einzelnen Delegationen unterschieden sich anfänglich stark hinsichtlich des Stellenwerts und der Reichweite einer potenziellen Bürgerschaft im Vertrag über die Europäische Union. Auffälligerweise waren es (neben dem EP) in erster Linie die kleinen und mittleren 
Mitgliedsstaaten, die sich für expansive Formulierungen einsetzten. ${ }^{8}$ Der maßgebende Vorschlag der spanischen Regierung etwa beinhaltete neben der Freizügigkeit und dem Lokalwahlrecht auch eine Verpflichtung der EU zur Wahrung der Chancengleichheit für alle BürgerInnen, Bürgerpflichten (z.B. Wehrpflicht) und die Möglichkeit zur Ausweitung des Geltungsbereichs auf BürgerInnen von Drittstaaten. Zur Begründung dieser weitreichenden Interpretation verknüpfte die Delegation funktionalistische Argumente, die an die Marktlogik der Primärinstitution pooled sovereignty anschlossen, mit einem Verweis auf Legitimitätskriterien im Kontext der Primärinstitutionen Demokratie und gemeinschaftliche Rechtsordnung. So würde durch die Unionsbürgerschaft einerseits der zur Umsetzung der Gemeinsamen Außen- und Sicherheitspolitik und der Wirtschafts- und Währungsunion nötige politische Raum geschaffen (Permanent Representation 1990). Gleichzeitig verstand sie diesen Raum als

"an integrated area serving the citizen, which will be the very source of democratic legitimacy and a fundamental pillar of the Union, through the progressive constitution of a common citizenship, the rights and obligations of which derive from the Union" (Délégation espagnole 1991).

Ein ähnliches framing findet sich im Bindi-Bericht des EP, der die Notwendigkeit einer Unionsbürgerschaft zum einen in funktionaler Logik als politische Folge der wirtschaftlichen Integration und Antwort auf Globalisierungsherausforderungen wie Migration, zum anderen als Bedingung für ein demokratisches Kräftegleichgewicht zwischen den Institutionen und den BürgerInnen der Union darstellte und sich dabei auf die ständige Rechtsprechung des EuGH bezog (Europäisches Parlament 1991: 4).

Dieses hybride Argumentationsmuster, wonach das erreichte Maß an Integration in einer Art normativem spillover nun Fragen demokratischer Legitimität nach sich ziehe, wurde somit zum dominanten framing der Bürgerschaftsfrage auf der Regierungskonferenz. Gleichzeitig war es zu unspezifisch, als dass es zur Legitimierung eines konkreten rechtlichen Rahmens dienen konnte. So konnte es auch von denjenigen VerhandlungsteilnehmerInnen übernommen werden, die einer Unionsbürgerschaft skeptisch gegenüberstanden. Dies ermöglichte ihnen, die normativen Argumente der BefürworterInnen anzunehmen, die Tragweite der Umsetzung in Sekundärinstitutionen jedoch zu beschränken. Der 1992 in Maastricht unterzeichnete Vertragstext blieb dementsprechend hinter den Erwartungen vieler IntegrationsbefürworterInnen zurück. Er gewährleistete zwar Freizügigkeit, das Wahlrecht im Wohnsitzland bei Kommunal- und EP-Wahlen, diplomatischen Schutz durch andere Mitgliedsstaaten in Drittländern und das Petitionsrecht beim EP, formulierte jedoch keine weitergehenden politischen oder sozialen Rechte. Auch BürgerInnenpflichten fanden keinen Einzug in den Vertrag. Der Vertrag beruft sich auf nationale Verfassungen als Quellen des Gemeinschaftsrechts (Art. F.) und verankert die Unionsbürgerschaft über die nationale Staatsbürgerschaft in den Mitgliedsstaaten (Art. 8.1).

8 O’Leary (1996: 23-30) bietet einen Überblick über die Positionen der einzelnen Delegationen. 
Er bekräftigt damit zwar die Primärinstitution der gemeinschaftlichen Rechtsordnung, bindet sie aber an ein konventionelles Völkerrechtsverständnis und den Grundsatz der nationalen Selbstbestimmung zurück. Offensichtlich drückt der Text damit nicht nur einen Kompromiss zwischen divergenten Verhandlungspositionen, sondern auch verschiedenen normativen Bezugspunkten aus.

\subsection{Die ASEAN Charta und Streitbeilegungsverfahren}

Die Unterzeichnung der ASEAN-Charta im Jahr 2007 begleitete einen politischen Prozess zum Aufbau der ASEAN Communities, der die Kooperation der Mitgliedsstaaten in einem sicherheitspolitischen, einem wirtschaftlichen und einem soziokulturellen Pfeiler verstärken sollte. Das Dokument markiert einen Schlüsselmoment in der rechtlichen Institutionalisierung der ASEAN - nicht nur, weil es der Organisation eine Rechtspersönlichkeit verlieh, sondern auch durch die Überarbeitung und Ausweitung ihrer Streitbeilegungsmechanismen. Bis dato hatten die ASEAN-Mitgliedsstaaten nichtbindende Formen der Streitbeilegung wie etwa vertrauensbildende Maßnahmen, Konsultationen und Mediation verfolgt (Caballero-Anthony 1998: 46-49). In der Praxis kamen darüber hinaus auch informelle diplomatische Mittel wie nichtöffentlicher peer pressure und strategische Anreize zum Einsatz (Acharya 2012b: 166). Im Gegensatz dazu sieht die Charta auch Zwangsmaßnahmen und verbindliche Schlichtungsprozesse vor.

Bereits vor der Charta hatten die ASEAN-Staaten mehrmals Versuche zur Stärkung institutioneller Konfliktlösung unternommen, doch diese blieben im Großen und Ganzen wirkungslos. 1976 verpflichteten sie sich durch die Unterzeichnung eines Friedens- und Freundschaftsvertrags (Treaty of Amity and Cooperation, TAC) auf friedliche Streitbeilegung. Das Abkommen sieht als freiwilligen Schlichtungsmechanismus einen Hohen Rat vor, der allerdings in der Praxis bedeutungslos blieb - keine Vertragsparte ${ }^{9}$ hat ihn bisher angerufen. Auch die Unterzeichnung prinzipiell bindender ASEAN-Wirtschaftsabkommen ab den späten 1970er-Jahren führte nicht zur Gründung unabhängiger Auslegungs- und Überwachungsorgane (EwingChow/Tan 2013: 5-6). 1992 wurde das ASEAN-Sekretariat mit dem monitoring des wirtschaftlichen Integrationsprozesses beauftragt, musste sich in dieser Rolle jedoch den Maßgaben der Mitgliedsstaaten unterordnen. Höchstes Entscheidungsgremium in Streitfragen blieb der intergouvernementale ASEAN-Gipfel (Lin 2010: 829). 1996 unterzeichneten die ASEAN-Staaten ein Protokoll zur Streitbeilegung bei der Verletzung von Wirtschaftsabkommen. Sie reagierten damit auf die Forderung von IndustrievertreterInnen nach mehr Rechtssicherheit bei wirtschaftlichen Aktivitäten in der Region. Allerdings verzichteten sie auf bindende Verfahren (McCall Smith 2000: 168). Das Protokoll sah lediglich Ad-hoc-Panels vor, die gute Dienste, Mediation und Schlichtung anbieten sollten und ausschließlich durch einen

9 Der Vertrag wurde 1987 für extraregionale Staaten geöffnet. Seitdem sind ihm 23 Staaten von außerhalb Südostasiens sowie die EU beigetreten. 
einstimmigen Beschluss der Mitgliedsstaaten eingesetzt werden konnten. Endgültige Entscheidungen wurden weiterhin von RegierungsvertreterInnen getroffen und beruhten dadurch auf politischem Interessenausgleich und nicht etwa juristischen Verfahren im engen Sinne.

Ende der 1990er-Jahre wurden Rufe nach einer grundlegenden Überarbeitung der Streitbeilegung lauter. Die beiden ASEAN-Generalsekretäre Rodolfo Severino (im Amt 1998-2003) und Ong Keng Yong (2003-2007) nutzten ihre informellen Agenda-Setting- und Koordinationskompetenzen, um wichtige Impulse zur rechtlichen Integration zu geben. So erklärte Severino (1998) bereits lange vor den eigentlichen Diskussionen um eine Charta:

"ASEAN may have to move toward the greater use of more formal instruments and binding commitments in the future, as developments like the financial and economic crisis [...] press ASEAN's members to ever closer coordination, cooperation and integration."

Aus dieser Diagnose leitete der Generalsekretär ein ehrgeiziges Reformprojekt $\mathrm{ab}$, in dem auch Durchsetzungsmechanismen und Schiedsgerichte eine wichtige Rolle spielten (Severino 2002). In dieser Initiative wurde er von IntegrationsbefürworterInnen aus ExpertInnenkommissionen, Beratungsfirmen und Think Tanks über die sogenannte Track-Two-Diplomatie unterstützt - einem System aus Konferenzen, Seminaren und Workshops für Policy-Dialoge, an denen RegierungsvertreterInnen in nichtoffizieller Kapazität teilnehmen. Auch wenn diese Diskursarenen letztendlich nicht völlig unabhängig von staatlichem Einfluss waren, konnten darin weitreichende Ideen zum Ausdruck gebracht werden. Eine von der ASEAN in Auftrag gegebene, einflussreiche McKinsey-Studie riet beispielsweise, die informellen Regeln der Organisation zu kodifizieren, ihr eine Rechtspersönlichkeit zu verleihen und die wirtschaftliche Integration durch starke Durchsetzungsmechanismen abzusichern (Schwarz/Villinger 2004: 45-46). Das Singapurer Institute for Southeast Asian Studies brachte sogar einen supranationalen Gerichtshof ins Spiel (Soesastro/Hew 2003: 294).

Die meisten dieser Vorschläge wurden in funktionalen frames begründet. Demnach führten Globalisierungsprozesse zu einem Wettbewerbsdruck, dem die südostasiatischen Volkswirtschaften am besten durch wirtschaftliche Integration standhalten könnten. Nur als starke Gemeinschaft würde ASEAN die Handels- und Sicherheitspolitik im weiteren asiatisch-pazifischen Raum mitgestalten und die Chancen nutzen können, die sich durch den Aufstieg Chinas und Indiens ergäben (Eminent Persons Group 2006: 14; Tay 2008: 155-156). Diese Ideen erhielten durch den noch frischen Eindruck der verheerenden Asienkrise der späten 1990er-Jahre einigen Nachdruck. Ein starker rechtlicher Rahmen für diesen Integrationsprozess erschien den eher liberal orientierten Akteuren daher als Schlüssel zu nachhaltigem Wachstum und regionaler Stabilität, insbesondere aufgrund der Abhängigkeit der Region von ausländischen Direktinvestitionen (Ewing-Chow/Tan 2013: 9-10, 14). Generalsekretär Severino (2001) betonte beispielsweise:

"closer regional economic integration requires basing it on binding legal foundations if integration is to be stable, credible and effective. The commitments undertaken must be clear, firm and enforceable [...]." 
Eine solche funktionalistische Spillover-Logik unterliegt auch den Think-TankAnalysen der Track-Two-Diplomatie und der erwähnten McKinsey-Studie (Schwarz/Villinger 2004; Soesastro/Hew 2003).

Diese Argumente blieben nicht wirkungslos, insbesondere nachdem die Regierungen der Mitgliedsstaaten im Oktober 2003 die Gründung einer ASEAN Economic Community vereinbart hatten. Die zur Planung der Wirtschaftsgemeinschaft beauftragte Kommission argumentierte prompt, dass die Organisation ihre Streitbeilegungsmechanismen nach dem Vorbild der World Trade Organization (WTO) reformieren müsse (High-Level Task Force 2003: Annex 1). Um sich gegen den erwarteten Anstieg der Streitfälle in einer stärker verflochtenen Wirtschaftsgemeinschaft zu rüsten, überarbeiteten die ASEAN-Regierungen das Streitschlichtungsprotokoll von 1996. Die Neuerungen zielten insbesondere darauf ab, die Einsetzung einer unabhängigen Berufungsinstanz zu erleichtern (Naldi 2014: 119-120). Funktionalistisches Denken zeigt sich auch im Vientiane Action Programme, in dem sich die Mitgliedsstaaten in Vorbereitung auf die ASEAN Communities zu Reformen bekennen und die Charta zum zentralen Instrument für das Gemeinschaftsprojekt erklären:

\footnotetext{
"Deepening integration, especially in the economic sphere, will also require the Member Countries to [...] make binding commitments, identify appropriate implementing timetables and mechanisms, extend national and regional capacities and competences, and develop institutional frameworks, responses and human resources in a range of areas that extend far beyond the existing scope of ASEAN integration" (ASEAN 2004, Section 5).
}

Dennoch kam es im zwischenstaatlichen Diskurs der ASEAN nicht zu einer vorbehaltlosen Übernahme funktionalistischer frames. Während die fünf Gründungsstaaten und Brunei den rechtlichen Integrationsprozess im Allgemeinen befürworteten, bekundeten die Regierungen der Ende der 1990er-Jahren beigetretenen CLMVGruppe - Kambodscha, Laos, Myanmar und Vietnam - offen ihre Skepsis gegenüber weitreichenden Änderungen (Chalermpalanupap 2009: 126; Koh 2009: passim). Ungeachtet ihrer zahlenmäßigen und wirtschaftlichen Unterlegenheit konnten die Regierungen dieser Staaten ihre Position durch eine günstige diskursive Opportunitätsstruktur legitimieren. Funktionalistischen Argumenten fehlte nämlich ein klarer normativer Bezugspunkt in den regionalen Primärinstitutionen. Im Gegensatz zur EG mit ihrem Pooled-Sovereignty-Paradigma war der ASEAN eine Marktlogik nicht von Beginn an eindeutig eingeschrieben. Trotz der weitgehenden Liberalisierung der südostasiatischen Volkswirtschaften wurden marktwirtschaftliche Prinzipien als regionale Kooperationsgrundlage zu sehr durch nationale Entwicklungsstrategien und Regimesicherungsbestrebungen relativiert, um uneingeschränkt als regionale Primärinstitution gelten zu können (Nesadurai 2003: 236-240).

Im Gegensatz dazu konnten die GegnerInnen einer weitreichenden Integration vier Primärinstitutionen mobilisieren, die die südostasiatische internationale Gesellschaft seit der Dekolonisierung geprägt hatten und die Grundlage für den ASEAN Way regionaler Kooperation bildeten. Wie in den frühen Jahren der EG beruhte die regionale Kooperation in Südostasien erstens auf einem traditionellen Völkerrechtsverständnis. Staaten galten dementsprechend als ausschließliche Träger legaler Autorität. Doch im Gegensatz zur EG, bei der die Widersprüche zwischen dem Prinzip 
der pooled sovereignty und dem zwischenstaatlichen Charakter der Rechtsordnung normativen Wandel ermöglichten, fehlten derlei Spannungen im primärinstitutionellen Kontext der ASEAN. ${ }^{10}$ Als zweite Primärinstitution wurde Souveränität nämlich in postkolonialer Tradition im Sinne einer strikten Nichteinmischungsnorm interpretiert. Sie war damit - drittens - voll kompatibel mit der Primärinstitution des Nationalismus und dem daraus abgeleiteten Selbstbestimmungsrecht der Völker. Und viertens wurde zwischenstaatliche Politik nach dem Prinzip der stillen Diplomatie geführt, die auf informellen Interessenausgleich statt öffentlichen Druck zielt (Acharya 2012b: 206; Haacke 2009: 3-7).

Auf diesen normativen Grundlagen konnten die CLMV-Regierungen das traditionelle ASEAN-Verständnis friedlicher Streitbeilegung verteidigen, das nicht nur die Abwesenheit von militärischer Gewalt, sondern auch einen allgemeinen Verzicht auf Zwangsmittel und ein Gebot gegenseitiger Verständigung im Konfliktmanagement beinhaltete (Caballero-Anthony 1998: 51-53). Schon als die Grundzüge der Charta diskutiert wurden, relativierte der damalige vietnamesische Premierminister Phan Van Khai (2004) die funktionalistische Logik unter Verweis auf die etablierten ASEAN-Normen:

"Globalization has increased interdependence among nations. A crisis or epidemic may
erupt in a country or region, but their impact could quickly spill over to other countries
and regions. [...] But we do not abuse regional cooperation to leverage pressure on other
countries or use it as an excuse to interfere into others' internal affairs. Thanks to this
unique 'ASEAN Way', many issues facing the Association have been addressed suc-
cessfully while ASEAN's non-interference principle continued to be upheld."

Khai nutzt hier den Begriff des ASEAN Way als Chiffre für die südostasiatischen Primärinstitutionen, um seine Präferenz für traditionelle Konfliktlösungspraktiken „tacit concultations, closed door diplomacy, and not rushing to regionalize or internationalize disagreements or disputes among nations“ - zu legitimieren. Um einer inkrementellen Aufweichung dieses Normkontexts zuvorzukommen, griffen RegierungsvertreterInnen der CLMV-Staaten auch die Debatte um Asiatische Werte auf. So erklärte Khai (2004):

"We believe that ASEAN fundamental principles will still be the foundation for the existence and development of our Association in the long run, even when the ASEAN Community is materialized [...]. I would like to emphasize the importance of culture in maintaining the relevance of ASEAN and its fundamental principles. They are reflective of Asian values [...]. They have been manifested as Asian way [sic!] in international relations $[\ldots] . "$ "

Letztendlich gelang den CLMV-Staaten dadurch eine Art "rhetorical entrapment" (Schimmelfennig 2001: 66). In ihrem framing erscheint jegliche weitreichende Reform unweigerlich als Abkehr von den konstitutiven Gemeinschaftsvorstellungen der ASEAN, wie auch Generalsekretär Severino (2002) eingestand. Diesen Eindruck wollten die reformorientierten Mitgliedsstaaten jedoch unbedingt vermeiden.

10 Auf das katalytische Potential von Spannungen zwischen Primärinstitutionen haben in der English School unter anderem Buzan (2004: 250-251) und Mayall (1990: passim) hingewiesen. 
In einer weniger kohärenten Opportunitätsstruktur hätten IntegrationsbefürworterInnen den Argumenten der konservativen Regierungen durch gegenläufige primärinstitutionelle Referenzen widersprechen können. So blieb ihnen nichts übrig, als das framing der CLMV-Staaten zu akzeptieren und dadurch deren Position zu legitimieren.

Dementsprechend hielt das Referenzdokument für die zur Vorbereitung der Charta-Verhandlungen gegründete Eminent Persons Group unmissverständlich fest:

„The ASEAN Charter will codify all ASEAN norms, rules, and values [and] reaffirm $[\ldots]$ the principles of inter-state relations in accordance with the UN Charter and established international law“ (ASEAN 2005).

Diese Rahmensetzungen schlossen eine weitreichende rechtliche Integration aus. Die ExpertInnen schlugen in ihrem Bericht zwar einige weitreichende Bestimmungen zur Sanktionierung von Verstößen sowie eine Klausel zum Verbot verfassungswidriger Regierungswechsel vor. Die eigens zum Entwurf der Charta eingesetzte High Level Task Force nahm diese jedoch nicht in den Vorschlag auf, den sie 2007 ihren Staats- und Regierungschefs vorlegten (Koh 2009: 60). Auch die Idee einer supranationalen Gerichtsinstanz wurde zwar diskutiert, letztendlich aber fallen gelassen (Woon 2009: 74).

Das Resultat dieser Umstände war eine Charta, deren völkerrechtliche Bedeutung von RechtsexpertInnen eher zurückhaltend bewertet wurde (Desierto 2011: 317-320; Lin 2010: 826, 836-837). Zwar fordert der Vertrag eine Ausweitung der bestehenden Streitbeilegungsmechanismen. Das zur Umsetzung dieser Bestimmung verabschiedete Protokoll behält den Mitgliedsstaaten aber weitgehende Freiheiten vor (Naldi 2014: 137). Statt eines ständigen Gerichtshofes bestimmt das Protokoll den ASEAN-Gipfel der Staats- und RegierungschefInnen als höchstes rechtliches Entscheidungsfindungs- und Durchsetzungsorgan. Da es keine Verfahrensregeln oder mögliche Sanktionsmaßnahmen spezifiziert, bleibt die Streitbeilegung in der ASEAN von politischen Maßgaben dominiert. Das Sekretariat erhielt unterdessen weitere Überwachungsfunktionen, aber kein Mandat zur Durchsetzung der ChartaVorschriften, sodass eine wirksame gerichtliche Aufsicht den nationalen Gerichten überlassen bleibt (Desierto 2011: 313-317; Ewing-Chow/Tan 2013: 16-17). Ein Anrufungsrecht für Privatpersonen ist nicht vorgesehen (Naldi 2014: 122), wodurch jegliche Idee einer ASEAN-Bürgerschaft im Keim erstickt wurde.

Der ehemalige Generalsekretär Rodolfo Severino (2007: 415-416) zeigte sich kurz nach der Unterzeichnung der Charta frustriert über die seiner Ansicht nach mangelnden Integrationsfortschritte. Die EntscheidungsträgerInnen der Gemeinschaft hätten nicht verstanden, dass sich ökonomische und politische Integration gegenseitig bedingen. In funktional-institutionalistischen Theorien lassen sich für die aus der Sicht von KritikerInnen halbherzige Verrechtlichung der ASEAN nur unbefriedigende Erklärungen finden. Zwar stimmt es, dass die interne Verflechtung der südostasiatischen Volkswirtschaften relativ schwach war. Eine konsequentere rechtliche Integration hätte jedoch auch den extern orientierten Handel und die globale Markteinbindung der ASEAN-Staaten gefördert. Aus streng rationalistischer Perspektive ist schwer nachzuvollziehen, warum die Regierungen zwar formal auf den Druck von 
wirtschaftlichen InteressenvertreterInnen reagierten, de facto aber einen zahnlosen Mechanismus schufen - zumal der Einfluss exportorientierter Industrieakteure auf politische Entscheidungsprozesse in den meisten südostasiatischen Staaten traditionell hoch ist (Solingen 2008). Die Entwicklungen sind vielmehr vor dem Hintergrund diskursiver Opportunitätsstrukturen zu verstehen, die einer weitführenden Integration entgegenstanden. Severino und seine MitstreiterInnen scheiterten nicht zuletzt daran, ihren Argumenten für eine effektive Verschiebung legaler Autorität von der nationalen auf die regionale Ebene normative Resonanz zu verleihen.

\section{Fazit}

Die empirischen Fallstudien von Verrechtlichungsprozessen in der EG/EU und der ASEAN bekräftigen die zu Beginn des Artikels aufgestellte These, dass regionale Integration maßgeblich von normativen Diskursen geprägt ist. In keinem der beiden Fälle haben politische AkteurInnen rechtliche Reformen einfach am Reißbrett als optimale Antworten auf funktionale Anforderungen entworfen oder in einem streng zweckrationalen Prozess ausgehandelt. Vielmehr legitimierten sie in langjährigen diskursiven Prozessen ihre institutionellen Präferenzen durch Einbettung in einen normativen Rahmen, um so deren Resonanz und Akzeptanz bei anderen DiskursteilnehmerInnen zu erhöhen und sie letztendlich im regionalen institution building durchzusetzen.

Jenseits dieses Plausibilitätstests zeigt eine komparative Interpretation der Fälle auch auf, wie eine Theorie normativer Diskurse unser Verständnis für die Grenzen klassischer Integrationstheorien erweitern kann, ohne dabei zentrale Konzepte wie das der funktionalistischen Integrationslogik aufzugeben. Sowohl in der EU als auch in der ASEAN führten IntegrationsbefürworterInnen nämlich funktionalistische Argumente für Verrechtlichungsschritte auf regionaler Ebene an. Während sie jedoch in Europa Spannungen in der primärinstitutionellen Opportunitätsstruktur (pooled sovereignty vs. Nationalismus) produktiv nutzen konnten und sich durch die Entwicklung zweier neuer Primärinstitutionen - Demokratie und gemeinschaftliche Rechtsordnung - legitimieren konnten, fehlte ein entsprechender Kontextwandel in Südostasien. Dies erleichterte den VerfechterInnen von Kooperation auf Basis des klassischen Völkerrechts die Delegitimierung funktionalistischer Argumente. Damit bestätigt die Analyse die eingangs formulierte Annahme, dass marktliberale und liberaldemokratische Primärinstitutionen eine günstige Opportunitätsstruktur für die Resonanz funktionalistischer frames bilden. Dabei war nicht ausschlaggebend, dass liberale Prinzipien in Europa zu einer transnationalen Öffentlichkeit geführt hätten, in der nichtstaatliche AkteurInnen ihre Interessen partizipativ zur Geltung hätten bringen können. Die zentralen normativen Diskurse blieben durchaus auf politische Eliten als primäre audiences beschränkt. Innerhalb dieser Diskursgemeinschaft erhöhten liberale Primärinstitutionen jedoch die Resonanz funktionalistischer Argumente, indem sie nichtstaatliche AkteurInnen als legitime Subjekte (als InhaberInnen von Rechten) und Objekte (als EmpfängerInnen 
öffentlicher Güter) einer transnationalen politischen Ordnung konstituierten, die dementsprechend rechtlich abzusichern sei.

Dieser Befund soll keinesfalls der Annahme Vorschub leisten, funktionalistische Integration sei ausschließlich in Europa möglich. Zum einen suggeriert bereits ein kursorischer Blick auf andere Sektoren wie etwa die handelspolitische Zusammenarbeit in der ASEAN Economic Community oder die finanzpolitische Chiang Mai Initiative, dass funktionalistische Argumente auch in Südostasien durchaus integrationsfördernd wirken können. Zum anderen war und ist auch in der Geschichte der EG/EU eine funktionalistische Logik nicht immer zentral. Das lässt sich schon in Stanley Hoffmanns (1966: 881-882, siehe auch Haas 1975: 6-8) Analyse der europäischen Integration in den 1960er-Jahren nachlesen, derzufolge eine staatszentriert-machtpolitische logic of diversity die marktorientierte logic of integration der Anfangsjahre abgelöst habe. In jüngerer Zeit hat der sich in nationalen Diskursen formierende backlash gegen einen scheinbar alternativlosen Integrationsautomatismus die Entwicklung einer dezidiert post-funktionalistischen Theorie befördert. Angesichts dessen lässt sich aus dem Vergleich kein universeller Befund zu regional dominanten Integrationslogiken ableiten.

Wohlgemerkt strebt eine Theorie normativer Diskurse nicht an, den Verlauf und die Resultate von Auseinandersetzungen auf der Mikroebene zu erklären, wie dies etwa der liberale Intergouvernementalismus für sich beansprucht. Welche Positionen in konkreten Verhandlungssituationen aufgrund spezifischer Geltungsansprüche Validität und Durchsetzungsmacht erhalten, lässt sich mittels praxisorientierter Ansätze sicherlich besser nachvollziehen (Adler-Nissen/Pouliot 2014; Niemann 2018). Die Stärke des hier entwickelten Analyseframeworks liegt vielmehr darin, dass es die normativen Bedingungen für die Legitimierung konkurrierender Ideen sichtbar macht. Der primärinstitutionelle Kontext von Integrationsdiskursen steckt konstitutiv den Möglichkeitshorizont für die darin vortragbaren Argumente ab. Durch die Ausmessung dieser Horizonte erlaubt die Theorie eine Spezifizierung der normativen scope conditions, unter denen bestimmte Integrationslogiken zum Tragen kommen. So verdeutlicht sie, dass und warum es funktionalistisch-institutionellen Theorien in manchen Kontexten an Plausibilität mangelt, etwa bezüglich der Responsivität der ASEAN gegenüber nationalen Interessenkoalitionen oder der schrittweisen Selbstlegitimierung des EuGH durch normatives Argumentieren. Indem der hier vertretene Ansatz Integrationslogiken als Motive normativer Diskurse endogenisiert, sie also von statischen Ursachen (causes) zu möglichen Gründen (reasons) für Integration umdeutet, ermöglicht er Theorientests im Sinne einer postfoundational Methodologie (Pouliot 2007: 364-374). Damit meine ich ein Vorgehen, das die Gültigkeit verschiedener in der Forschungsliteratur vertretener Interpretationen von Integrationsprozessen nicht durch die Überprüfung kausaler Hypothesen bestimmt, sondern anhand der tatsächlichen Diskurse bemisst, die in zeitlich, räumlich und sektoral begrenzten Prozessen regionale Integration bedingen. Auf dieser Grundlage können dann gegebenenfalls in einem zweiten Schritt konkrete Entscheidungen mithilfe klassischer Ansätze erklärt werden. 
Trotz oder gerade wegen eines solchen empirischen groundings lässt der Ansatz durchaus Generalisierungen und feinkörnigere Hypothesen zu, die die Theoriediskussion in der Vergleichenden Regionalismusforschung besonders durch die enge Zusammenführung von Strukturs- und Akteursperspektive bereichern können. Über die Erforschung der Resonanzbedingungen funktionalistischer frames hinaus drängen sich Fragen für die weiterführende Forschung auf. Welche Primärinstitutionen eignen sich besonders zur Legitimierung einer intergouvernementalen BargainingLogik? In welchen Politikbereichen haben regionale Integrationsprozesse postkoloniale Normvorstellungen im Globalen Süden besonders weit zugunsten einer liberalen Governance-Rationalität verdrängt, und wie verhandeln regionale Organisationen Spannungen zwischen diesen beiden Logiken? Führt der weltweite Aufstieg populistischer Regime zur bloßen Ablehnung von Regionalismus oder entwickeln regionale populistische Allianzen eigene Argumentationsmuster zur Rechtfertigung spezifischer Kooperationsformen? Ohne hier bereits eine systematische Forschungsagenda zu entwerfen, ist dieser Artikel doch ein klares Votum dafür, dass ein kritischer Dialog zwischen Integrations- und Regionalismusforschung einen wichtigen Beitrag zur Entwicklung einer globalen IB-Forschung leisten kann.

\section{Literatur}

Acharya, Amitav 2009: Whose Ideas Matter? Agency and Power in Asian Regionalism, Ithaca, NY.

Acharya, Amitav 2012a: Comparative Regionalism. A Field whose Time has Come? In: The International Spectator 47: 1, 3-15.

Acharya, Amitav 2012b: The Making of Southeast Asia. International Relations of a Region, Singapur.

Acharya, Amitav 2016: Regionalism beyond EU-Centrism, in: Börzel, Tanja A./Risse, Thomas (Hrsg.): The Oxford Handbook of Comparative Regionalism, Oxford, 109-130.

Ad-hoc-Ausschuss für das Europa der Bürger 1985: Bericht des Ad-hoc-Ausschusses für das Europa der Bürger an den Europäischen Rat in Brüssel am 29. und 30. März 1985, in: Bulletin der Europäischen Gemeinschaften 3, 128-134.

Adler-Nissen, R./Pouliot, Vincent 2014: Power in Practice. Negotiating the International Intervention in Libya, in: European Journal of International relations 20: 4, 889-911.

Alter, Karen J. 2009: Who are the >Masters of the Treaty ? European Governments and the European Court of Justice, in Dies.: The European Court's Political Power. Selected Essays, Oxford, 109-136.

ASEAN 2004: Vientiane Action Programme 2004-2010 (Vientiane, 29. November), in: https:// www.asean.org/storage/images/archive/VAP-10th\%20ASEAN\%20Summit.pdf; 29.8.2019.

ASEAN 2005: Kuala Lumpur Declaration on the Establishment of the ASEAN Charter (Kuala Lumpur, 5. Dezember), in: https://asean.org/asean/asean-charter/kuala-lumpur-declaratio $\mathrm{n} / ; 29.8 .2019$.

Bieling, Hans-Jürgen/Lerch, Marika (Hrsg.) 2012: Theorien der europäischen Integration., Wiesbaden.

Börzel, Tanja A. 2016: Theorizing Regionalism: Cooperation, Integration, and Governance, in: Börzel, Tanja A./Risse, Thomas (Hrsg.): The Oxford Handbook of Comparative Regionalism, Oxford, 41-63. 
Börzel, Tanja A./Risse, Thomas (Hrsg.) 2012: From Europeanisation to Diffusion. West European Politics Sonderheft 35: 1.

Bull, Hedley 1977: The Anarchical Society. A Study of Order in World Politics, London.

Burley, Anne-Marie/Mattli, Walter 1993: Europe before the Court. A Political Theory of Legal Integration, in: International Organization 47: 1, 41-76.

Buzan, Barry 2004: From International to World Society? English School Theory and the Social Structure of Globalisation, Cambridge.

Buzan, Barry 2009: The Middle East through English School Theory, in: Buzan, Barry/Gonzalez-Pelaez, Ana (Hrsg.). International Society and the Middle East: English School Theory at the Regional Level, Basingstoke, 24-44.

Caballero-Anthony, Mely 1998: Mechanisms of Dispute Settlement. The ASEAN Experience, in: Contemporary Southeast Asia 20: 1, 38-66.

Chalermpalanupap, Termsak 2009: In Defence of the ASEAN Charter, in: Koh, Tommy/ Manalo, Rosario G./Woon, Walter (Hrsg.): The Making of the ASEAN Charter, Singapur, 117-135.

Checkel, Jeffrey T. 2016: Regional Identities and Communities, in: Börzel, Tanja A./Risse, Thomas (Hrsg.): The Oxford Handbook of Comparative Regionalism, Oxford, 559-578.

Deitelhoff, Nicole 2009: The Discursive Process of Legalization. Charting Islands of Persuasion in the ICC Case, in: International Organization 63: 1, 33-65.

Deitelhoff, Nicole/Müller, Harald 2005: Theoretical Paradise - Empirically Lost? Arguing with Habermas, in: Review of International Studies 31: 1, 167-179.

Délégation espagnole 1991: Note à la Conférence intergouvernementale sur l'Union politique. Objet: Citoyenneté européene (CONF-UP 1731/91, Brüssel, 20. Februar).

Desierto, Diane A. 2011: ASEAN's Constitutionalization of International Law. Challenges to Evolution under the New ASEAN Charter, in: Columbia Journal of Transnational Law 49: 2, 268-320.

Diez, Thomas 1996: Postmoderne und europäische Integration. Die Dominanz des Staatsmodells, die Verantwortung gegenüber dem Anderen un die Konstruktion eines alternativen Horizonts, in: Zeitschrift für Internationale Beziehungen 3: 2, 255-281.

Diez, Thomas 1999: Die EU lesen. Diskursive Knotenpunkte in der britischen Europadebatte, Opladen.

Diez, Thomas/Manners, Ian/Whitman, Richard G. 2011: The Changing Nature of International Institutions in Europe. The Challenge of the European Union, in: Journal of European Integration 33: 2, 117-138.

Eminent Persons Group on the ASEAN Charter 2006: Report, in: http://www.asean.org/wp-co ntent/uploads/images/archive/19247.pdf; 29.8.2018.

European Parliament 1979: Proceedings of the Round Table on >Special Rights and a Charter of the Rights of the Citizens of the European Community< and Related Documents (Florence, 26-28 October), Luxembourg.

Europäische Kommission 1988: Europa der Bürger (Mitteilung der Kommission an das Europäische Parlament, KOM (88) 331 endg., 7. Juli).

Europäischer Rat 1990: Schlussfolgerungen des Vorsitzes (SN/304/2/90), in: Europäisches Parlament - Tätigkeiten Sonderausgabe 3/S-90, 1-12.

Europäisches Parlament 1984: Entwurf eines Vertrages zur Gründung der Europäischen Union, in: Amtsblatt EG 1984 Serie C.

Europäisches Parlament 1991: Zwischenbericht des Institutionellen Ausschusses über die Unionsbürgerschaft (Bindi-Bericht, PE DOK A3-0139/91 und PE 150.034/endg.), in: Europäisches Parlament: Sitzungsdokumente, 23. Mai.

Ewing-Chow, Michael/Tan, Hsien-li 2013: The Role of the Rule of Law in ASEAN Integration (EUI Working Papers RSCAS 2013/16), San Domenico di Fiesole.

Finnemore, Martha/Toope, Stephen J. 2001: Alternatives to >Legalization<. Richer Views of Law and Politics, in: International Organization 55: 3, 743-758. 
Garrett, Geoffrey 1992: International Cooperation and Institutional Choice. The European Community's Internal Market, in: International Organization 46: 2, 533-560.

George, Alexander L./Bennett, Andrew 2005: Case Studies and Theory Development, Cambridge, MA.

Grieco, Joseph 1997: Systemic Sources of Variation in Regional Institutionalization in Western Europe, East Asia, and the Americas, in Milner, Helen/Mansfield, Edward (Hrsg.): The Political Economy of Regionalism, New York, NY.

Haas, Ernst B. 1958: The Uniting of Europe. Political, Social and Economic Forces 1950-1957, Stanford, CA.

Haas, Ernst B. 1961: International Integration. The European and the Universal Process, in: International Organization 15: 3, 366-392.

Haas, Ernst B. 1975: The Obsolescence of Regional Integration Theory (Institute of International Studies Research Series, No. 25), Berkeley, CA.

Haltern, Ulrich 2004: Integration through Law, in: Diez, Thomas/Wiener, Antje (Hrsg.): European Integration Theory, Oxford, 177-196.

Hartmann, Christof 2016: Sub-Saharan Africa, in: Börzel, Tanja A./Risse, Thomas (Hrsg.): The Oxford Handbook of Comparative Regionalism, Oxford, 271-294.

Hemmer, C./Katzenstein, Peter J. 2002: Why is there no NATO in Asia? Collective Identity, Regionalism, and the Origins of Multilateralism., in: International Organization 56: 3, 575-607.

Higgott, Richard 2014: Alternative Models of Regional Cooperation? The Limits of Regional Institutionalization in East Asia., in: Telò, Mario (Hrsg.): European Union and New Regionalism, Farnham, 87-114.

High-Level Task Force on ASEAN Economic Integration 2003: Recommendations, in: http://as ean.org/?static post=recommendations-of-the-high-level-taskforce-on-asean-economic-i ntegration; $17 . \overline{5} .2018$.

Hoffman, Stanley 1966: Obstinate or Obsolete? The Fate of the Nation-State and the Case of Western Europe, in: Daedalus 95: 3, 862-915.

Holzinger, Katharina 2001: Kommunikationsmodi und Handlungstypen in den Internationalen Beziehungen. Anmerkungen zu einigen irreführenden Dichotomien, in: Zeitschrift für Internationale Beziehungen 8: 2, 243-286.

Hooghe, Liesbet/Marks, Gary 2009: A Postfunctionalist Theory of European Integration. From Permissive Consensus to Constraining Dissensus, in: British Journal of Politics and International Relations 39: 1, 1-23.

Hooghe, Liesbet/Marks, Gary 2019 (Hrsg.): Grand Theories of European Integration in the 21st Century, in: Journal of European Public Policy Sonderheft (online first), DOI: 10.1080/13501763.2019.1569711.

Jachtenfuchs, Markus/Diez, Thomas/Jung, Sabine 1998: Which Europe? Conflicting Models of a Legitimate European Political Order, in: European Journal of International Relations 4: 4, 409-445.

Jetschke, Anja 2017: What Drives Institutional Reforms in Regional Organisations? Diffusion, Contextual Conditions, and the Modular Design of ASEAN, in: TRaNS: Trans-Regional and -National Studies of Southeast Asia 5: 1, 173-196.

Jetschke, Anja/Katada, Saori N. 2016: Asia, in Börzel, Tanja A./Risse, Thomas (Hrsg.): The Oxford Handbook of Comparative Regionalism, Oxford, 225-248.

Joerges, Christian/Neyer, Jürgen 1997: From International Bargaining to Deliberative Political Processes. The Constitutionalisation of Comitology, in: European Law Journal 3: 3, 273-299.

Kanthak, Leon 2012: Explaining Differences in the Institutional Design of ASEAN and NAFTA., in: Börzel, Tanja A./Goltermann, Lukas/Striebinger, Kai (Hrsg.): Roads to Regionalism. Genesis, Design, and Effects of Regional Organizations, Farnham, 81-99.

Katzenstein, Peter J. 2005: A World of Regions. Asia and Europe in the American Imperium, Ithaca, NY. 
Khai, Phan Van 2004: ASEAN Lecture (Hanoi, 8. August), in: http://www.asean.org/asean-le cture-by-he-mr-prime-minister-phan-van-khai-ha-noi/; 29.8.2019.

Knudsen, Tonny Brems 2018: Fundamental Institutions and International Organizations. Theorizing Continuity and Change, in: Knudsen, Tonny Brems/Navari, Cornelia (Hrsg.): International Organization in International Society. The Institutional Structure of World Order, Cham. 23-50.

Koh, Tommy 2009: The Negotiating Process, in: Koh, Tommy/Manalo, Rosario G./Woon, Walter (Hrsg.): The Making of the ASEAN Charter, Singapore, 47-68.

Koopmans, Ruud/Olzak, Susan 2004: Discursive Opportunities and the Evolution of RightWing Violence in Germany, in: American Journal of Sociology 110: 1, 198-230.

Kornprobst, Markus 2011: The Agent's Logics of Action. Defining and Mapping Political Judgement, in: International Theory 3: 1, 70-104.

Korte, Annika 2012: Why Did NAFTA and ASEAN Set up Dispute Settlement Procedures? In: Börzel, Tanja A./Goltermann, Lukas/Striebinger, Kai (Hrsg.): Roads to Regionalism. Genesis, Design, and Effects of Regional Organizations, Farnham, 101-115.

Krapohl, Sebastian/Dinkel, Julia/Faude, Benjamin 2010: Judicial Integration in the Americas? A Comparison of Dispute Settlement in NAFTA and MERCOSUR, in: Laursen, Finn (Hrsg.): Comparative Regional Integration. Europe and beyond, Abingdon, 169-192.

Krebs, Ronald R./Jackson, Patrick Thaddeus 2007: Twisting Tongues and Twisting Arms. The Power of Political Rethoric, in: European Journal of International Relations 13: 1, 35-66.

Legal Affairs Committee 1979: Opinion (Bayerl Report), in: Proceedings of the Round Table on >Special Rights and a Charter of the Rights of the Citizens of the European Community< and Related Documents (Flocence, 26 to 28 October), Luxembourg, 84-92.

Lenz, Tobias/Marks, Gary 2016: Regional Institutional Design, in: Börzel, Tanja A./Risse, Thomas (Hrsg.): The Oxford Handbook of Comparative Regionalism, Oxford, 513-537.

Levi Sandri, Lionello 1961: Intervention, in: European Parliament: Debates of the European Parliament of 22 November 1961 on Social Harmonisation, 134-142.

Lin, Chung Hun 2010: ASEAN Charter. Deeper Regional Integration under International Law, in: Chinese Journal of International Law 9: 4, 821-837.

Maas, Willem 2007: Creating European Citizens, Lanham, MD.

Mattli, Walter 1999: The Logic of Regional Integration. Europe and beyond, Cambridge.

Mattli, Walter/Slaughter, Anne-Marie 1998: Revisiting the European Court of Justice, in: International Organization 52: 1, 177-209.

Mayall, James 1990: Natioanlism and International Society, Cambridge.

McCall Smith, James 2000: The Politics of Dispute Settlement Design. Explaining Legalism in Regional Trade Pacts, in: International Organization 54: 1, 137-180.

Meyer, Christoph 1999: Political Legitimacy and the Invisibility of Politics. Exploring the European Union's Communication Deficit, in: Journal of Common Market Studies 37: 4, 617-639.

Milward, Alan 1992: The European Rescue of the Nation-State, London.

Moravcsik, Andrew 1998: The Choice for Europe. Social Purpose and State Power from Messina to Maastricht. Ithaca, NY.

Murau, Steffen/Spandler, Kilian 2016: EU, US and ASEAN Actorness in G20 Financial Policy Making. Bridging the EU Studies-New Regionalism Divide, in: Journal of Common Market Studies 54: 4, 928-943.

Murray, Philomena 2010: Comparative Regional Integration in the EU and East Asia. Moving beyond Integration Snobbery, in: International Politics 47: 3-4, 308-23.

Müller, Harald 1994: Internationale Beziehungen als kommunikatives Handeln: Zur Kritik der utilitaristischen Handlungstheorien, in: Zeitschrift für Internationale Beziehungen 1: 1, 15-44.

Naldi, Gino J. 2014: The ASEAN Protocol on Dispute Settlement Mechanisms. An Appraisal, in: Journal of International Dispute Settlement 5: 1, 105-138. 
Nesadurai, Helen E. S. 2003: Attempting Developmental Regionalism through AFTA. The Domestic Sources of Regional Governance, in: Third World Quarterly 24: 2, 235-253.

Niemann, Arne 2006: Explaining Decisions in the European Union, Cambridge.

Niemann, Holger 2018: Praktiken der Rechtfertigung im UN-Sicherheitsrat. Von der Konstitution zur Koordination normativer Ordnungen durch das Veto, in: Zeitschrift für Internationale Beziehungen 25: 1, 36-64.

O'Leary, Siofra 1996: The Evolving Concept of Community Citizenship. From the Free Movement of Persons to Union Citizenship, London.

Permanent Representation of Spain to the European Communities 1990: The Road to European Citizenship (Council Document 9223/90, Annex 7, 24. September).

Phelan, William 2018: European Legal Integration. Towards a More Liberal Intergovernmentalist Approach, in: Journal of Common Market Studies 56: 7, 1562-1577.

Pouliot, Vincent 2007: >Sobjectivism〈. Toward a Constructivist Methodology, in: International Studies Quarterly 51: 2, 359-384.

Pouliot, Vincent 2008: The Logic of Practicality. A Theory of Practice of Security Communities, in: International Organization 62: 2, 257-288.

Ragin, Charles C. 1987: The Comparative Method. Moving beyond Qualitative and Quantitative Strategies, Berkeley, CA.

Risse-Kappen, Thomas 1995: Reden ist nicht billig. Zur Debatte um Kommunikation und Rationalität, in: Zeitschrift für Internationale Beziehungen 2: 1, 171-184.

Risse-Kappen, Thomas 1996: Exploring the Nature of the Beast. International Relations Theory and Comparative Policy Analysis Meet the European Union, in: Journal of Common Market Studies 34: 1, 53-80.

Risse, Thomas 2000: >Let's Argue!^ Communicative Action in World Politics, in: International Organization 54: 1, 1-39.

Rittberger, Berthold/Schimmelfennig Frank (Hrsg.) 2006: Die Europäische Union auf dem Weg in den Verfassungsstaat, Frankfurt a.M.

Sbragia, Alberta 2008: Comparative Regionalism. What Might it Be? In: The JCMS Annual Review of the European Union in 2007, 29-49.

Sbragia, Alberta/Söderbaum, Frederik 2010: EU Studies and the ^New Regionalism can be Gained from Dialogue? In: Journal of Common Market Studies 32: 6, 563-582.

Schimmelfennig, Frank 1997: Rhetorisches Handeln in der internationalen Politik, in: Zeitschrift für Internationale Beziehungen 4: 2, 219-254.

Schimmelfennig, Frank 2001: The Community Trap. Liberal Norms, Rhetorical Action, and the Eastern Enlargement of the European Union, in: International Organization 55: 1, 47-80.

Schimmelfennig, Frank 2002: Liberal Community and Enlargement. An Event History Analysis, in: Journal of European Public Policy 9: 4, 598-626.

Schimmelfennig, Frank 2003: Osterweiterung. Strategisches Handeln und kollektive Ideen, in: Jachtenfuchs, Markus/Kohler-Koch, Beate (Hrsg.): Europäische Integration, Wiesbaden, 541-568.

Schimmelfennig, Frank 2012: Zwischen Neo- und Postfunktionalismus. Die Integrationstheorien und die Eurokrise, in: Politische Vierteljahresschrift 53: 3, 394-413.

Schimmelfennig, Frank 2018: Regional Integration Theory, in: Oxford Research Encyclopedia of Politics. DOI: 10.1093/acrefore/9780190228637.013.599.

Schmitter, Philippe C. 1969: Three Neo-Functional Hypotheses about International Integration, in: International Organization 23: 1, 161-166.

Schwarz, Adam/Villinger, Roland 2004: Integrating Southeast Asia's Economies, in: McKinsey Quarterly 1, 36-47.

Seidendorf, Stefan 2006: Geschichtlichkeit und Gemeinschaftsumwelt. Was strukturiert den Konstitutionalisierungsprozess? In: Rittberger, Berthold/Schimmelfennig, Frank (Hrsg.): Die Europäische Union auf dem Weg zum Verfassungsstaat, Frankfurt a.M., 101-138. 
Severino, Rodolfo C. 1998: Asia Policy Lecture. What ASEAN is and what it Stands for (Sydney, 22. Oktober), in: https://asean.org/?static_post=asia-policy-lecture-what-asean-is-an d-what-it-stands-for-by-rodolfo-c-severino-secretary-general-of-asean-at-the-research-in stitute-for-asia-and-the-pacific-university-of-sydney-australia-22-october-1; 29.8.2019.

Severino, Rodolfo C. 2001: The ASEAN Way and the Rule of Law (Address at the International Law Conference on ASEAN Legal Systems and Regional Integration, Kuala Lumpur, 3. September), in: https://asean.org/?static_post=the-asean-way-and-the-rule-of-la w; 29.8.2019.

Severino, Rodolfo C. 2002: The Three Ages of ASEAN (Lecture Delivered at the ARCO Forum of Public Affairs, Cambridge, MA, 3. Oktober), in: https://asean.org/?static_post=th e-three-ages-of-asean; 29.8.2019.

Severino, Rodolfo C. 2007: ASEAN beyond Forty. Towards Poltical and Economic Integration, in: Contemporary Southeast Asia 29: 3, 406-423.

Soesastro, Hadi/Hew, Denis 2003: Realizing the ASEAN Economic Community by 2020. ISEAS and ASEAN-ISIS Approaches, in: ASEAN Economic Bulletin 20: 3, 292-296.

Solingen, Etel 2008: The Genesis, Design and Effects of Regional Institutions. Lessons from East Asia and the Middle East, International Studies Quarterly 52: 1, 261-294.

Spandler, Kilian 2015: The Political International Society. Change in Primary and Secondary Institutions, in: Review of International Studies 41: 3, 601-622.

Spandler, Kilian 2018a: Regional Organizations in International Society. ASEAN, the EU and the Politics of Normative Arguing, Cham.

Spandler, Kilian 2018b: Regional Standards of Membership and Enlargement in the EU and ASEAN, in: Asia Europe Journal 16: 2, 183-198.

Stone Sweet, Alec/Sandholtz, Wayne 1997: European Integration and Supranational Governance, in: Journal of European Public Policy, 4: 3, 297-317.

Söderbaum, Frederik 2004: Modes of Regional Governance in Africa. Neoliberalism, Sovereignty-Boosting and Shadow Networks, in: Global Governance 10: 4, 419-436.

Söderbaum, Frederik 2016: Rethinking Regionalism, London.

Tay, Simon S. C. 2008: The ASEAN Charter. Between National Sovereignty and the Region's Constitutional Moment, in: Singapore Yearbook of International Law 12, 151-170.

Thomas, Daniel C. 2017: Beyond Identity. Membership Norms and Regional Organization, in: Journal of European Public Policy 13: 8, 1190-210.

Van Langenhove, Luk 2011: Building Regions. The Regionalization of World Order, Farnham.

Warleigh-Lack, Alex 2006: Towards a Conceptual Framework for Regionalisation. Bridging >New Regionalism< and >Integration Theory<, in: Review of International Political Economy 13: $5,750-771$.

Wheeler, Nicholas J. 2000: Saving Strangers: Humanitarian Intervention in International Society, Oxford.

Wiener, Antje 1998: >European< Citizenship Practice. Building Institutions of a Non-State, Boulder, CA.

Wiener, Antje/Börzel, Tanja A./Risse, Thomas (Hrsg.) 2018: European Integration Theory, Oxford.

Woon, Walter 2009: The ASEAN Charter Dispute Settlement Mechanisms, in: Koh, Tommy/ Manalo, Rosario G./Woon, Walter (Hrsg.): The Making of the ASEAN Charter, Singapore, 69-77.

Zhang, Yonghin 2015: Regional International Society in East Asia? A Critical Investigation, in: Global Discourse 5: 3, 360-373. 